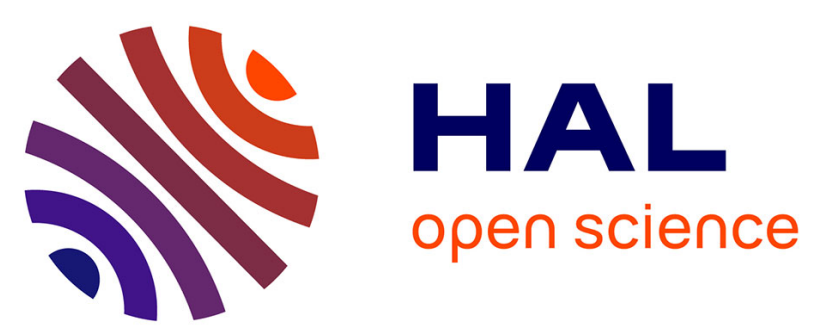

\title{
Singularity spectrum of fractal signals from wavelet analysis: Exact results
}

\author{
Emmanuel Bacry, Jean-François Muzy, Alain Arneodo
}

\section{To cite this version:}

Emmanuel Bacry, Jean-François Muzy, Alain Arneodo. Singularity spectrum of fractal signals from wavelet analysis: Exact results. Journal of Statistical Physics, 1993, 70 (3-4), pp.635-674. 10.1007/bf01053588 . hal-01557137

\section{HAL Id: hal-01557137 \\ https://hal.science/hal-01557137}

Submitted on 5 Jul 2017

HAL is a multi-disciplinary open access archive for the deposit and dissemination of scientific research documents, whether they are published or not. The documents may come from teaching and research institutions in France or abroad, or from public or private research centers.
L'archive ouverte pluridisciplinaire HAL, est destinée au dépôt et à la diffusion de documents scientifiques de niveau recherche, publiés ou non, émanant des établissements d'enseignement et de recherche français ou étrangers, des laboratoires publics ou privés. 


\title{
Singularity Spectrum of Fractal Signals from Wavelet Analysis: Exact Results
}

\author{
E. Bacry, ${ }^{1,2}$ J. F. Muzy, ${ }^{1}$ and A. Arnéodo ${ }^{1}$
}

The multifractal formalism for singular measures is revisited using the wavelet transform. For Bernoulli invariant measures of some expanding Markov maps, the generalized fractal dimensions are proved to be transition points for the scaling exponents of some partition functions defined from the wavelet transform modulus maxima. The generalization of this formalism to fractal signals is established for the class of distribution functions of these singular invariant measures. It is demonstrated that the Hausdorff dimension $D(h)$ of the set of singularities of Hölder exponent $h$ can be directly determined from the wavelet transform modulus maxima. The singularity spectrum so obtained is shown to be not disturbed by the presence, in the signal, of a superimposed polynomial behavior of order $n$, provided one uses an analyzing wavelet that possesses at least $N>n$ vanishing moments. However, it is shown that a $C^{\infty}$ behavior generally induces a phase transition in the $D(h)$ singularity spectrum that somewhat masks the weakest singularities. This phase transition actually depends on the number $N$ of vanishing moments of the analyzing wavelet; its observation is emphasized as a reliable experimental test for the existence of nonsingular behavior in the considered signal. These theoretical results are illustrated with numerical examples. They are likely to be valid for a large class of fractal functions as suggested by recent applications to fractional Brownian motions and turbulent velocity signals.

KEY WORDS: Multifractal formalism; invariant measures; fractal signals; Hölder exponents; wavelet analysis; wavelet transform modulus maxima.

\section{INTRODUCTION}

Fractal and multifractal concepts ${ }^{(1-3)}$ are now widely used in a variety of physical situations. ${ }^{(4-6)}$ In its present form, the multifractal approach is essentially adapted to describe the statistical scaling properties of singular

\footnotetext{
${ }^{1}$ Centre de Recherche Paul Pascal, Avenue Schweitzer, 33600 Pessac, France.

${ }^{2}$ Permanent address: Ecole Normale Supérieure, 75230 Paris Cedex, France.
} 
measures. ${ }^{(2,7-12)}$ Notable examples of such measures are the invariant probability measure of a dynamical system, ${ }^{(2,3,9-12)}$ the harmonic measure of a diffusion-limited aggregate, ${ }^{(4,6,13-15)}$ the distribution of a voltage drop across a random resistor network, ${ }^{(4,13,14)}$ and the spatial distribution of the dissipation field of fully developed turbulence. ${ }^{(3,16-18)}$ The multifractal formalism $^{(2)}$ involves decomposing fractal measures into intervowen sets, each of which is characterized by its singularity strength $\alpha$ and its Hausdorff dimension $f(\alpha)$. The so-called $f(\alpha)$ singularity spectrum was shown to be intimately related to the "generalized" fractal dimensions ${ }^{(19-21)}$ $D_{q}=\tau(q) /(q-1)$ that can be extracted from the power-law behavior of the partition function (in the limit $l \rightarrow 0^{+}$)

$$
Z_{q}=\sum_{i} \mu_{i}^{q}(l) \sim l^{\tau(q)}
$$

where the sum is over disjoint intervals of size $l$ and $\mu_{i}(l)$ is the measure contained in each interval. Actually, there exists a deep analogy that links the multifractal formalism with that of statistical thermodynamics. ${ }^{(10-12,22-24)}$ The variables $q$ and $\tau(q)$ play the same role as the inverse of temperature and the free energy in thermodynamics, ${ }^{(25)}$ while the Legendre transform

$$
f(\alpha)=\min _{q}[q \alpha-\tau(q)]
$$

indicates that instead of the energy and the entropy, we have $\alpha$ and $f(\alpha)$ as the thermodynamic variables conjugate to $q$ and $\tau(q)$. This thermodynamic multifractal formalism has been worked out in mathematics in the context of dynamical systems theory. ${ }^{(22-24)}$ But rigorous proofs of the above connection have been only limited to some restricted classes of singular measures, e.g., invariant measures of some expanding Markov maps ("cookie cutter" Cantor sets) on an interval or a circle,${ }^{(9,10)}$ the invariant measure associated to the dynamical systems for period doubling and for critical circle mappings with golden rotation number. ${ }^{(10)}$ It has recently been developed into a powerful technique accessible also to experimentalists. Successful applications have been reported for multifractal measures which appear beyond the scope of dynamical systems. ${ }^{(6)}$

In several phenomena, fractals appear not only as singular measures, but as singular functions. ${ }^{(1,13,14)}$ The examples range from plots of various kinds of random walks to interfaces developing in reaction-limited growth processes and to turbulent velocity signals at inertial range scales. ${ }^{(6)}$ There have been several attempts to extend the concept of multifractality to singular functions. ${ }^{(26,27)}$ In the context of fully developed turbulence, the 
multiscaling properties of the turbulent velocity signal were investigated by calculating the moments of the probability density function of (longitudinal) velocity increments ${ }^{(26,28-30)}$

$$
\delta v_{l}(x)=v(x+l)-v(x)
$$

over inertial separation $l$. By Legendre transforming the scaling exponents $\zeta_{p}$ of the structure functions of order $p$

$$
S_{p}(l)=\left\langle\delta v_{l}^{p}\right\rangle \sim l^{\zeta_{p}}
$$

one gets the Hausdorff dimension $D(h)$ of the subset of $\mathbb{R}$ for which velocity increments behave as $l^{h}$. In a more general context, $D(h)$ will be the spectrum of Hölder exponents for the singular signal under study and thus will have a similar status as the $f(\alpha)$ singularity spectrum for singular measures. But there are some fundamental limitations to the structure function approach, which intrinsically fails to fully characterize the $D(h)$ singularity spectrum. ${ }^{(31,32)}$ Even though one can extend this method from integer to real positive $p$ values by considering an absolute value on velocity increments ${ }^{(27)}$ in Eq. (4), the structure functions generally do not exist for $p<0$, since there is no reason a priori that the probability density function of $\delta v_{l}$ vanishes for $\delta v_{l}=0$. Thus, only the strongest singularities are amenable to the velocity structure function method and only partial information on the $D(h)$ spectrum (actually its increasing left-hand part) can be extracted. Moreover, the measurement of the contribution of Hölder exponents $h<1$ (singularities in the first derivative of the signai) can be dramatically disturbed by the presence of regular behavior as well as the existence of weakest singularities with $h>1$ (singularities in higher derivatives). In ref. 33 we systematically reviewed the intrinsic insufficiencies of the structure function method. Even though this method was a first interesting step toward a multifractal theory of singular functions, this theory is still lacking and there is still a need for an appropriate powerful technical tool to deal with fractal functions.

In previous work ${ }^{(31-33)}$ we elaborated on a novel strategy which is likely to provide a practical way to determine this entire singularity spectrum $D(h)$ directly from any experimental signal. This approach is essentially based on the use of a mathematical tool introduced in signal analysis in the early 1980s: the wavelet transform. ${ }^{(34-38)}$ The wavelet transform has been recently emphasized as a very efficient technique to collect microscopic information about the scaling properties of multifractal measures. ${ }^{(39,40)}$ Extensive applications to various multifractal measures, including the invariant measures of some well-known discrete dynamical systems, have clearly demonstrated its fascinating ability to reveal the 
hierarchy that governs the spatial distribution of the singularities. ${ }^{(39-44)}$ What makes the wavelet transform such an attractive tool in the present study is that its singularity scanning ability equally applies to singular signals as to singular measures. ${ }^{(31-33,40,45-47)}$

Indeed, the simplest way of performing a multifractal analysis of a singular measure ${ }^{(2)}$ is to partition it using boxes of size $l$. Then the measure in each box of size $l$ can be characterized by a singularity strength $\alpha$ by $\mu_{i}(l) \sim l^{\alpha_{i}}$, where the index $i$ denotes the box number. The number of occurrences of a particular value $\alpha$ defines the $f(\alpha)$ singularity spectrum by $N(\alpha) \sim l^{-f(\alpha)}$. A wavelet can actually be seen as an oscillatory variant of a characteristic function of a box (i.e., a "square" function). Thus, one can generalize in a rather natural way the multifractal formalism to singular functions by defining some partition functions in terms of the wavelet coefficients. ${ }^{(31-33)}$ Let us note that by choosing a wavelet which is orthogonal to polynomial behavior up to order $N$, one can make the wavelet transform blind to the regular behavior of a function, ${ }^{(31-33,40)}$ remedying in this way one of the main difficulties inherent to the dramatic failure of the structure function method [note that the square function is the primitive of what has been called the "poor man's wavelet": $\psi(x)=\delta(x-1)-\delta(x)$, implicitly used in the increment method and which is only orthogonal to constants]. Then, from the Legendre transform of the scaling exponents $\tau(q)$ of these wavelet-based partition functions, one can extract the whole $D(h)$ spectrum of Hölder exponents. Indeed, at a given scale, instead of using a continuous integral over space [like the increment method in Eq. (4)], we sum discretely over the local maxima of the wavelet transform ${ }^{(47)}$ so that we remove divergencies for $q<0$ and also incorporate the multiplicative structure (if there is any) of the singularity distribution directly into the calculation of the partition function. ${ }^{(31,32)}$ Each connected line of local maxima is likely to emanate from a singularity of the signal ${ }^{(47)}$ Along these maxima lines, the wavelet transform behaves at small scales $^{(47)}$ as a power law with an exponent $h(x)$ which is equal to the Hölder exponent of the signal at the point $x$. The number of such lines at a certain scale $a$ and corresponding to the same $h$ defines the $D(h)$ spectrum of Hölder exponents by $N(h) \sim a^{-D(h)}$ (when $a$ goes to zero). Moreover, one can further proceed to an adaptive scale partitioning which consists in maximizing (or minimizing according to the sign of $q$ ) the partition function by choosing the optimal wavelet covering. In this spirit, the free energy $\tau(q)$ is obtained as a transition point for the scaling exponent of some partition function and the entropy $D(h)$ can be shown to be the Hausdorff dimension of the set of singularities of Hölder exponent $h$.

In our previous work ${ }^{(31,32)}$ we have outlined the main trends of our multifractal approach of singular functions based on wavelets and shown 
some successful applications to fractional Brownian motions and fully developed turbulence data. Our purpose here is to give rigorous proofs of the validity of this formalism when applied to a self-affine function which is the distribution function of a Bernoulli measure lying on a (disconnected) Cantor set invariant under some affine Markov map. We also derive exact results when this class of self-affine functions is perturbed by a polynomial or a general $C^{\infty}$ behavior. We comment on the possibility of extending this mathematical analysis to a larger class of singular functions, with the ultimate goal of establishing the foundations of a universal multifractal theory of singular distributions including singular measures and singular functions.

The paper is organized as follows. Section 2 contains some background material on the (one-dimensional) continuous wavelet transform. We define the wavelet transform modulus maxima ${ }^{(47)}$ and the notion of maxima lines. In Section 3, we revisit the multifractal formalism for singular measures using the wavelet decomposition. We give rigorous proofs that the generalized fractal dimensions [and consequently the $f(\alpha)$ spectrum by Legendre transforming $\left.\tau(q)=(q-1) D_{q}\right]$ of Bernoulli invariant measures of some expanding linear Markov maps can be obtained as transition points for the scaling exponents of some partition functions defined either from the continuous wavelet transform or from the wavelet transform maxima lines only. In Section 4, we prove that, when using the latter approach, these results naturally extend to self-affine functions that are distribution functions of the above multifractal Bernoulii measures. We demonstrate that the Hausdorff dimension $D(h)$ of the set of singularities of Hölder exponent $h$ can be directly determined from the wavelet transform maxima lines. We also prove that the determination of this singularity spectrum is not disturbed by the presence of polynomial behavior of order $n$, provided one uses an analyzing wavelet that possesses at least $N>n$ vanishing moments. This is no longer true when considering any $C^{\infty}$ perturbation; it generally results in a nonanalyticity of the $D(h)$ singularity spectrum which displays a phase transition ${ }^{(12,48-51)}$ that masks the weakest singularities. This phase transition is shown to depend on the number $N$ of vanishing moments of the analyzing wavelet. The rigorous proof of the existence of this phase transition is rather technical and requires some additional hypothesis. Section 5 is devoted to numerical applications which illustrate that our theoretical results are valid for noncompact support analyzing wavelets which decrease fast enough at infinity, and for a larger class of self-affine functions including some functions that are almost everywhere singular. We conclude in Section 6. 


\section{THE WAVELET TRANSFORM}

The wavelet transform of a function $f$ according to the analyzing wavelet $\psi$ is defined as ${ }^{(34-38)}$

$$
T_{\psi}[f](b, a)=\frac{1}{a} \int_{-\infty}^{\infty} \bar{\psi}\left(\frac{x-b}{a}\right) f(x) d x
$$

where $a \in \mathbb{R}^{+*}$ and $b \in \mathbb{R}$. Generally $\psi$ is chosen to be localized in both space and frequency, so that $T_{\psi}$ can be seen as an accurate space-frequency analysis ( $b$ is the space parameter and $1 / a$ is the frequency parameter). Moreover, $\psi$ is usually chosen to be orthogonal to polynomials, so that $T_{\psi}[f]$ can be used to "detect" the singularities of $f$. Indeed, in order to detect a singular behavior such as $x^{\alpha+n}(n \in \mathbb{N}$ and $\alpha \in] 0,1[$, which is masked by a regular behavior $P_{n}(x)$ [where $P_{n}(x)$ is a polynomial of degree smaller than $n+1], \psi$ has to satisfy ${ }^{(40,45)} \int x^{k} \psi(x) d x=0$ for $0 \leqslant k \leqslant n$.

For our purpose, we will mainly assume the following:

(H1) $\psi$ is a real-valued function which has a compact support $(\subset]-1 / 2,1 / 2[$ ).

(H2) $\psi$ is $C^{2}$.

If (H1) is replaced by $\psi(x)=O\left(x^{-k}\right), \forall k>0$, similar theorems are obtained, but the proofs are more complicated. To make the point of this paper as clear as possible, we have chosen to use a compactly supported analyzing wavelet.

In the same way we have defined the wavelet transform of a function in Eq. (5), we can extend the wavelet transform to a measure $\mu,{ }^{(39-44)}$

$$
T_{\psi}[\mu](b, a)=\int_{\text {Supp } \mu} \psi\left(\frac{x-b}{a}\right) d \mu(x)
$$

where Supp $\mu$ is the support of $\mu$. Notice that, in this definition, we have omitted the $1 / a$ normalization factor which appears in Eq. (5), so that

$$
T_{\psi}[\mu](b, a)=-T_{\psi}[f](b, a)
$$

where $\psi^{\prime}$ denotes the first derivative of $\psi$ and $f(x)=\int_{0}^{x} d \mu(x)$. Equation (7) will allow us to derive results about functions (Section 4) from results about measures (Section 3). For the sake of simplicity, $T_{\psi}[\mu](b, a)$ and $T_{\psi}[f](b, a)$ will be referred to as $T_{\psi}(b, a)$.

We need two more definitions (cf. ref. 47]).

Definition 1. $(x, a) \in \mathbb{R} \times \mathbb{R}^{+*}$ [sometimes referred to as $x(a)$ ] will be said to be a modulus maximum of the wavelet transform $T_{\psi}$ iff

$$
\exists \varepsilon \in \mathbb{R}^{+*}, \quad \forall x_{1} \in \mathbb{R}, \quad 0<\left|x_{1}-x\right|<\varepsilon \Rightarrow\left|T_{\psi}\left(x_{1}, a\right)\right|<\left|T_{\psi}(x, a)\right|
$$


If $(x, a)$ is a modulus maximum, then

$$
\frac{\partial T_{\psi}}{\partial b}(x, a)=0
$$

Definition 2. We call a maxima line at scale $a_{0}(>0)$ of $T_{\psi}$ any connected curve $l$ in the $(b, a)$ half-plane so that:

(a) $(x, a) \in l \Rightarrow a \leqslant a_{0}$ and $(x, a)$ is a modulus maximum of $T_{\psi}$.

(b) $\forall a, 0<a \leqslant a_{0} \Rightarrow \exists x \in \mathbb{R} /(x, a) \in l$.

Notation 1. $\mathscr{L}\left(a_{0}\right)$ will denote the set of all the maxima lines at scale $a_{0}$ and $\mathscr{L}$ the set of all the maxima lines (at any scale).

Notation 2. If $l \in \mathscr{L}\left(a_{0}\right)$ and if $a \leqslant a_{0}$, then $l l_{a}$ will denote the set $\left\{\left(x, a^{\prime}\right) \in l / a^{\prime} \leqslant a\right\}$.

Notation 3. If $l \in \mathscr{L}, a_{l}$ will denote the maximum scale $a$ which satisfies $\exists l_{1} \in \mathscr{L}(a), l \subset l_{1}$.

Notation 4. For the sake of simplicity, if $I$ is a subset of $\mathbb{R}$, we will (improperly) write $l \subset I$ whenever for any $(x, a)$ in $l, x$ belongs to $I$.

\section{DETERMINING THE GENERALIZED FRACTAL DIMENSIONS OF A MEASURE FROM ITS WAVELET TRANSFORM}

\subsection{The Dynamical System}

Let us consider the expanding piecewise linear maps $T$ on $A=[0,1]$ for which $T^{-1}(A)$ is a finite union of disjoint intervals

$$
T^{-1}(A)=\bigcup_{i=1}^{s} A_{i}
$$

Let $g$ be the smallest gap between two consecutive intervals, i.e.,

$$
g=\min _{i}\left\{\operatorname{dist}\left(A_{i}, A_{i+1}\right)\right\}
$$

We then define

$$
\begin{aligned}
T_{i}^{-1}: & A \rightarrow A_{i} \\
& x \rightarrow T_{i}^{-1}(x)=T^{-1}(x)=\lambda_{i} x+\beta_{i}
\end{aligned}
$$

where $0<\lambda_{i}<1$. Let $A=\inf _{i} \lambda_{i}$. We also set

$$
A_{k_{1}, \ldots, k_{n}}=A \cap T_{k_{1}}^{-1}(A) \cap T_{k_{2}}^{-1} \circ T_{k_{1}}^{-1}(A) \cdots \cap T_{k_{n}}^{-1} \circ \cdots \circ T_{k_{1}}^{-1}(A)
$$


Then it is clear that if $J$ denotes the invariant set under the mapping $T, J$ is the limit of the set (when $n \rightarrow+\infty$ )

$$
\mathscr{A}^{(n)}=A \cap T^{-1}(A) \cdots \cap T^{-n}(A)=\bigcup_{\substack{k_{i}=1 \ldots s \\ i=1 \cdots n}} A_{k_{1}, \ldots, k_{n}}
$$

i.e., $J$ can be written as

$$
J=\bigcap_{n}^{\infty} \mathscr{A}^{(n)}
$$

The mapping $T$ is a linear version of more general one-dimensional mappings usually referred to as "cookie cutters"(9) or expanding Markov maps. ${ }^{(10)}$ To this mapping one can associate a family of invariant measures called the Bernoulli measures associated to $T$. A Bernoulli measure is a measure $\mu$ which satisfies $\left.\exists\left(p_{1}, \ldots, p_{s}\right) \in\right] 0,1\left[{ }^{s}, \sum_{i} p_{i}=1\right.$, so that

$$
\forall\left(k_{1}, \ldots, k_{n}\right) \in\{1, \ldots, s\}^{n}, \quad \mu\left(A_{k_{1} \cdots k_{n}}\right)=p_{k_{1}} \cdots p_{k_{n}}
$$

The generalized fractal dimensions $D_{q}(q \in \mathbb{R})$ of such a measure ${ }^{(2,19-21)}$ are then given by the following equation:

$$
D(q)=\frac{\tau(q)}{q-1}
$$

where $\tau(q)$ is obtained by solving

$$
\sum_{i=1}^{s} \frac{p_{i}^{q}}{\lambda_{i}^{\tau(q)}}=1
$$

The $f(\alpha)$ singularity spectrum ${ }^{(2,9,10)}$ is then obtained by Legendre transforming $\tau(q)$,

$$
f(\alpha)=\min _{q}(\alpha q-\tau(q))
$$

In the following section, the measures $\mu$ we consider are Bernoulli measures of $T$ which have a gap $g$ [Eq. (9)] different from 0 . The set of such measures will be called $\mathscr{M}$.

\subsection{Exact Results}

In the following, for any interval $I, I(a)$ will denote the same interval "extended" in both directions by $a / 2$, i.e., $I(a)=\{I+a / 2\} \cup\{I-a / 2\}$.

Let $\mu \in \mathscr{M}$ (i.e., $\mu$ is a Bernoulli measure with $g>0$ ). 
Lemma 1. Local self-similarity of the wavelet transform, $\forall a$, $0<a<g, \forall b \in]-\infty, \infty[$ :

(i) $b \notin A(a) \Rightarrow T_{\psi}(b, a)=0$.

(ii) $b \in A(a) \Rightarrow \forall k \in\{1, \ldots, s\}, T_{\psi}(b, a)=\left(1 / p_{k}\right) T_{\psi}\left(T_{k}^{-1}(b), \lambda_{k} a\right)$.

Proof. Let us distinguish the two cases:

(i) $b \notin A(a)$. Then, $T_{\psi}(b, a)=0$ follows easily from the fact that $\psi$ has a compact support in $]-1 / 2,1 / 2[$ [cf. (H1)] and that Supp $\mu \subset A$.

(ii) $b \in A(a)$. Let $k \in\{1, \ldots, s\}$; then, by setting $x^{\prime}=T_{k}^{-1}(x)$ one obtains

$$
T_{\psi}(b, a)=\int_{A} \psi\left(\frac{x-b}{a}\right) d \mu(x)=\frac{1}{p_{k}} \int_{A_{k}} \psi\left(\frac{T_{k}(x)-b}{a}\right) d \mu(x)
$$

and by using Eq. (10) we get

$$
T_{\psi}(b, a)=\frac{1}{p_{k}} \int_{A_{k}} \psi\left(\frac{x-T_{k}^{-1}(b)}{\lambda_{k} a}\right) d \mu(x)
$$

As $T_{k}^{-1}(b) \in A_{k}\left(\lambda_{k} a\right)$ and $\lambda_{k} a<a<g$, therefore, if $x \in A_{k^{\prime}}$ with $k \neq k^{\prime}$, then $\left|x-T_{k}^{-1}(b)\right|>g / 2$. It follows that

$$
T_{\psi}(b, a)=\frac{1}{p_{k}} \int_{A} \psi\left(\frac{x-T_{k}^{-1}(b)}{\lambda_{k} a}\right) d \mu(x)=\frac{1}{p_{k}} T_{\psi}\left(T_{k}^{-1}(b), \lambda_{k} a\right)
$$

3.2.1. Extracting $\tau(q)$ from the Continuous Wavelet Transform. Let $\mu \in \mathscr{M}$; we then introduce the following partition function for $q \geqslant 1$ and $p \in \mathbb{R}$ (see refs. 40,52 , and 53):

$$
\begin{aligned}
K_{p, q}: & \mathbb{R}^{+*} \rightarrow \mathbb{R}^{+} \\
& a \rightarrow K_{p, q}(a)=a^{-p} \int_{\mathbb{R}}\left|T_{\psi}(b, a)\right|^{q-1} d b
\end{aligned}
$$

Lemma 2. Renormalization of $K_{p, q}(a): \forall p \in \mathbb{R}, \forall q \geqslant 1, \forall a<\Lambda g$,

$$
K_{p, q}(a)=\sum_{k=1}^{s} \frac{p_{k}^{q-1}}{\lambda_{k}^{p-1}} K_{p, q}\left(\frac{a}{\lambda_{k}}\right)
$$

Proof. Let $a<\Lambda g$. From (H1) and from the fact that Supp $\mu \subset\left(\bigcup_{i} A_{i}\right)$, it follows that $\left.T_{\psi}(\cdot, a)\right|_{\left(\cup_{i} A_{i}(a)\right)^{r}}=0$; therefore

$$
K_{0, q}(a)=\int_{\mathbb{R}}\left|T_{\psi}(b, a)\right|^{q-1} d b=\sum_{k=1}^{s} \int_{A_{k}(a)}\left|T_{\psi}(b, a)\right|^{q-1} d b
$$


Then, by setting $b=T_{k}^{-1}\left(b^{\prime}\right)$ [Eq. (10)],

$$
K_{0, q}(a)=\sum_{k=1}^{s} \int_{A\left(a / \lambda_{k}\right)} \lambda_{k}\left|T_{\psi}\left(T_{k}^{-1}(b), a\right)\right|^{q-1} d b
$$

and by using Lemma 1(ii),

$$
K_{0, q}(a)=\sum_{k=1}^{s} \int_{A\left(a / \lambda_{k}\right)} p_{k}^{q-1} \lambda_{k}\left|T_{\psi}\left(b, \frac{a}{\lambda_{k}}\right)\right|^{q-1} d b
$$

Moreover, for $b \notin A\left(a / \lambda_{k}\right)$, by using Lemma 1(i) $\left(a / \lambda_{k}<g\right)$, we get $T_{\psi}\left(b, a / \lambda_{k}\right)=0$. It follows that

$$
K_{0, q}(a)=\sum_{k=1}^{s} \int_{\mathbb{R}} p_{k}^{q-1} \lambda_{k}\left|T_{\psi}\left(b, \frac{a}{\lambda_{k}}\right)\right|^{q-1} d b=\sum_{k=1}^{s} p_{k}^{q-1} \lambda_{k} K_{0, q}\left(\frac{a}{\lambda_{k}}\right)
$$

Finally, by multiplying both sides by $a^{-p}$, we obtain Eq. (19).

In order to get the main theorem [i.e., how to extract the $D_{q}$ from $\left.K_{p, q}(a)\right]$, we need to study the class of functions which satisfy a renormalization relation like Eq. (19). The following lemma was first stated in ref. 40; we reproduce its proof in Appendix A.

Lemma 3. Let $f: \mathbb{R}^{+} \rightarrow \mathbb{R}^{+}$that satisfies $\exists s \in \mathbb{N}, \exists\left(c_{1}, \ldots, c_{s}\right) \in$ $\left.\left.\left(\mathbb{R}^{+*}\right)^{s}, \exists\left(\lambda_{1}, \ldots, \lambda_{s}\right) \in\right] 0,1\right]^{s}$, and $\exists g>0$ so that

$$
0<a<g \Rightarrow f(a)=\sum_{k=1}^{s} c_{k} f\left(\frac{a}{\lambda_{k}}\right)
$$

Then, the following two relations hold:

(i) If $\forall \varepsilon, 0<\varepsilon<g$, $\sup _{[\varepsilon, g]} f(x)<+\infty$, then

$$
\sum_{k} c_{k}<1 \Leftrightarrow \lim _{a \rightarrow 0^{+}} f(a)=0
$$

(ii) If $\forall \varepsilon, 0<\varepsilon<g$, inf $[\varepsilon, g] f(x)>0$, then

$$
\sum_{k} c_{k}>1 \Leftrightarrow \lim _{a \rightarrow 0^{+}} f(a)=+\infty
$$

By applying this lemma for $f=K_{p, q}$, we get a method to recover $\tau(q)$ from $K_{p, q}$, for $q \geqslant 1$.

Theorem 1. Let $\mu \in \mathscr{M}$. Let $K_{p, q}(a)$ be its corresponding partition function defined in Eq. (18) and let $D_{q}=\tau(q) /(q-1)$ be its generalized 
fractal dimensions [Eqs. (15) and (16)]. Then, for $q \geqslant 1, \tau(q)$ is the transition exponent, so that

$$
\begin{aligned}
& p-1<\tau(q-1) \Rightarrow \lim _{a \rightarrow 0^{+}} K_{p, q}(a)=0 \\
& p-1>\tau(q-1) \Rightarrow \lim _{a \rightarrow 0^{+}} K_{p, q}(a)=+\infty
\end{aligned}
$$

Remark. For a general analyzing wavelet $\psi$, it seems unlikely that a similar theorem would hold for $q \leqslant 1$. Indeed, it seems difficult to "control" the parts of $T_{\psi}$ which are close to 0 for any kind of $\psi$. In the very particular case where $\psi$ is a "nice" strictly positive function, it has been shown in ref. 53 that a theorem similar to Theorem 1 holds for all $q$; but, as we will see in Section 4.2 , in order to extract the singularity spectrum of a function, it is fundamental to estimate $\tau(q)$ (for all $q$ ) using an analyzing wavelet with vanishing moments ${ }^{(40,45)}$ (and therefore which oscillates around 0 ).

3.2.2. Extracting $\tau(q)$ from the Wavelet Transform Modulus Maxima. The main idea is that, instead of defining a partition function by using at each scale $a$ all the values $T_{\psi}(b, a)(b \in \mathbb{R})$, we will only use the values of $T_{\psi}$ at its modulus maxima ${ }^{(31,32)}$ (see Definition 1). One could define the partition function $Z_{p, q}(a)=\sum_{i} a^{-p}\left|T_{\psi}\left(x_{i}(a), a\right)\right|^{q}$ (where $\left\{x_{i}(a)\right\}_{i}$ are the modulus maxima at scale $a$ ); even though it is very easy to show that $Z_{p, q}$ satisfies a renormalization property [like Eq. (19)], we still do not have any control on $\sup _{[\varepsilon, g]} Z$ (for $q<0$ ) as required by Lemma 3(i). In order to circumvent this difficulty, instead of using the values of $T_{\psi}$ at the modulus maxima, we are going to use the maximum values of $\left|T_{\psi}\right|$ along the maxima lines (see Definition 2).

Let us make two additional mild hypotheses. We suppose that:

(H3) $\exists a>0$ so that $\mathscr{L}(a) \neq \varnothing$.

(H4) $\forall a>A g, \mathscr{L}(a)$ is a finite set.

These hypotheses allow us to define the following partition function (for $q \in \mathbb{R})$ :

$$
\begin{aligned}
Z_{p, q}: & \mathbb{R}^{+*} \rightarrow \mathbb{R}^{+} \\
& a \rightarrow Z_{p, q}(a)=a^{-p} \sum_{l \in \mathscr{L}(a)}\left(\sup _{\left(x, a^{\prime}\right) \in l}\left|T_{\psi}\left(x, a^{\prime}\right)\right|\right)^{q}
\end{aligned}
$$

Remark. Let us note that this definition is more or less equivalent to choosing an "optimal" covering of the support of $\mu$ (using different scales) which maximizes (for $q \geqslant 0$ ) or minimizes (for $q<0$ ) the partition function. 
This is very close to the original definition of the partition function described in ref. 2 .

From now on $\widetilde{T}_{k}$ and $\widetilde{T}_{k}^{-1}(k \in\{1, \ldots, s\})$ will denote the following $(b, a)$ half-plane maps:

$$
\begin{aligned}
\tilde{T}_{k}(b, a) & =\left(T_{k}(b), a / \lambda_{k}\right) \\
\tilde{T}_{k}^{-1}(b, a) & =\left(T_{k}^{-1}(b), a \lambda_{k}\right)
\end{aligned}
$$

Lemma 4. Renormalization of $Z_{p, q}: \forall(p, q) \in \mathbb{R}^{2}, \forall a<A g$,

$$
Z_{p, q}(a)=\sum_{k=1}^{s} \frac{p_{k}^{q}}{\lambda_{k}^{p}} Z_{p, q}\left(\frac{a}{\lambda_{k}}\right)
$$

Proof. (a) Let $l \in \mathscr{L}(a), a<g$. From Lemma 1(i) and the fact that $l$ is a set of modulus maxima, it follows that $l \subset A(a)$ (see Notation 4). Therefore, by using Lemma 1 (ii), $\forall k \in\{1, \ldots, s\}, \forall\left(x, a^{\prime}\right) \in l$,

$$
T_{\psi}\left(x, a^{\prime}\right)=\frac{1}{p_{k}} T_{\psi}\left(\widetilde{T}_{k}^{-1}\left(x, a^{\prime}\right)\right)
$$

and then $l_{k}=\tilde{T}_{k}^{-1}(l) \in \mathscr{L}\left(\lambda_{k} a\right)$. Moreover, from the definition of $\tilde{T}_{k}^{-1}$, $l_{k} \subset A_{k}(a)$. It then follows that any maxima line at scale $a$ corresponds to $s$ distinct maxima lines $\left\{l_{k}\right\}_{k \in\{1, \ldots, s\}}$, respectively, at scale $\lambda_{k} a$ and included in $A_{k}(a)$.

(b) Conversely, let $l \in \mathscr{L}(a), a<\Lambda g$. From (H1) and the fact that supp $\mu \subset \bigcup_{i} A_{i}$, it follows that $\left.T_{\psi}\right|_{\left(\cup_{i} A_{i}(a)\right)^{c}}=0$. Then it is clear that $\exists ! j \in\{1, \ldots, s\}$, so that $l \subset A_{j}(a)$. Then, by using Lemma $1($ ii $), \forall\left(x, a^{\prime}\right) \in l$

$$
T_{\psi}\left(x, a^{\prime}\right)=p_{j} T_{\psi}\left(\widetilde{T}_{j}\left(x, a^{\prime}\right)\right)
$$

from which one deduces that $\tilde{T}_{j}(l) \in \mathscr{L}\left(a / \lambda_{j}\right)$. Thus, any maxima line at scale $a$ corresponds to a unique maxima line at one of the scales $\left\{a / \lambda_{k}\right\}_{k \in\{1, \ldots, s\}}$.

From these two results, one can get easily that if $a<A g, \mathscr{L}(a)$ can be decomposed into $s$ disjoint subsets $\left\{\mathscr{L}_{k}(a)\right\}_{k \in\{1, \ldots, s\}}$, where $\mathscr{L}_{k}(a)=\left\{l \in \mathscr{L}(a), l \subset A_{k}(a)\right\}$. Moreover, $\mathscr{L}_{k}(a)=\widetilde{T}_{k}^{-1}\left(\mathscr{L}\left(a / \lambda_{k}\right)\right)$. Therefore

$$
\begin{aligned}
Z_{0, q}(a) & =\sum_{l \in \mathscr{L}_{(a)}}\left(\sup _{l}\left|T_{\psi}\right|\right)^{q}=\sum_{k=1}^{s} \sum_{l \in \mathscr{L}_{k}(a)}\left(\sup _{l}\left|T_{\psi}\right|\right)^{q} \\
& =\sum_{k=1}^{s} \sum_{l \in \tilde{T}_{k}^{-1}\left(\mathscr{L}\left(a / \lambda_{k}\right)\right)}\left(\sup _{l}\left|T_{\psi}\right|\right)^{q}=\sum_{k=1}^{s} \sum_{l \in \mathscr{L}_{\left(a / \lambda_{k}\right)}}\left(\sup _{l}\left|T_{\psi} \circ \tilde{T}_{k}^{-1}\right|\right)^{q}
\end{aligned}
$$


Then, by using Lemma 1(ii),

$$
Z_{0, q}(a)=\sum_{k=1}^{s} \sum_{l \in \mathscr{\mathscr { L }}\left(a / \lambda_{k}\right)} p_{k}^{q}\left(\sup _{l}\left|T_{\psi}\right|\right)^{q}=\sum_{k=1}^{s} p_{k}^{q} Z_{0, q}\left(\frac{a}{\lambda_{k}}\right)
$$

Equation (22) follows by multiplying this relation by $a^{-p}$.

In order to apply Lemma 3 to $f=Z_{p, q}$, we need to prove that $\forall q \in \mathbb{R}$, $\forall \varepsilon \in \mathbb{R}^{+*}, \sup _{[\varepsilon, A g]} Z_{p, q}<\infty$ and $\inf _{[\varepsilon, A g]} Z_{p, q}>0$. Obviously, the hard part is to prove that $\sup _{[\varepsilon, A g]} Z_{p, q} \neq \infty$ for $q<0$. For that purpose, we need to find a lower bound for $\sup _{l}\left|T_{\psi}\right|[\forall l \in \mathscr{L}(a)]$. that:

Lemma 5. $\forall a<A g, \forall l \in \mathscr{L}(a), \exists ! n \in \mathbb{N}, \exists !\left(k_{1}, \ldots, k_{n}\right) \in\{1, \ldots, s\}^{n}$ so

(a) $\tilde{T}_{k_{1}} \cdots \widetilde{T}_{k_{n}}(l) \in \mathscr{L}\left(a /\left(\lambda_{k_{1}} \cdots \lambda_{k_{n}}\right)\right)$

(b) $a /\left(\lambda_{k_{1}} \cdots \lambda_{k_{n-1}}\right) \leqslant \Lambda g<a /\left(\lambda_{k_{1}} \cdots \lambda_{k_{n}}\right)$.

Moreover, the following equation holds:

$$
\left.T_{\psi}\right|_{l}=\left.p_{k_{1}} \cdots p_{k_{n}} T_{\psi} \circ \widetilde{T}_{k_{n}} \circ \cdots \circ \widetilde{T}_{k_{1}}\right|_{l}
$$

Proof. We proved in the proof of Lemma 4(b) that $\forall l \in \mathscr{L}(a)$ $(a<\Lambda g), \exists ! j \in\{1, \ldots, s\}$ so that $\widetilde{T}_{j}(l) \in \mathscr{L}\left(a / \lambda_{j}\right)$. Moreover, we saw that for this particular $j,\left.T_{\psi}\right|_{l}=\left.p_{j} T_{\psi} \circ \tilde{T}_{j}\right|_{l}$. By applying this result several times, $j$ takes successively the values $k_{1}, k_{2}, \ldots$, until $a /\left(\lambda_{k_{1}} \cdots \lambda_{k_{n}}\right)$ becomes greater than $A g$.

Lemma 6. $\exists C \in \mathbb{R}^{+*}$ so that

$$
\forall a<A g, \quad \forall l \in \mathscr{L}(a) \quad \sup _{l}\left|T_{\psi}\right| \geqslant C a^{\alpha_{\max }}
$$

where $\alpha_{\max }=\sup _{i}\left(\ln p_{i} / \ln \lambda_{i}\right)$

Proof. It is clear that $\forall l \in \mathscr{L}(a) \quad(a>\Lambda g), \exists C_{l}>0$ so that $\sup _{\left(x, a^{\prime}\right) \in l}\left|T_{\psi}\left(x, a^{\prime}\right)\right|>C_{l}$. From (H4) it follows that there exists a strictly positive constant $C_{1}$ so that

$$
C_{1}=\inf _{a>\Delta g, l \in \mathscr{L}_{(a)}} C_{l}
$$

Now let $l \in \mathscr{L}(a)(a<\Lambda g)$ and let $k_{1}, \ldots, k_{n}$ given by Lemma 5 . Let $l_{n}=\tilde{T}_{k_{n}} \ldots \widetilde{T}_{k_{1}}(l)\left[l_{n} \in \mathscr{L}\left(a_{0}\right)\right.$ with $\left.a_{0}>\Lambda g\right]$. We then obtain 


$$
\begin{aligned}
& \sup _{\left(x, a^{\prime}\right) \in l}\left|T_{\psi}\left(x, a^{\prime}\right)\right|=p_{k_{1}} \cdots p_{k_{n}} \sup _{\left(x, a^{\prime}\right) \in l_{n}}\left|T_{\psi}\left(x, a^{\prime}\right)\right| \geqslant C_{l_{n}} p_{k_{1}} \cdots p_{k_{n}} \\
& \geqslant C_{1} \lambda_{k_{1}}^{\ln p_{k_{1}} / \ln \lambda_{k_{1}}} \ldots \lambda_{k_{n}}^{\ln p_{k_{n}} / \ln \lambda_{k_{n}}} \\
& \geqslant C_{1}\left(\lambda_{k_{1}} \cdots \lambda_{k_{n}}\right)^{\sup _{i}\left(\ln p_{i} / \ln \lambda_{i}\right)} \\
& \geqslant C_{1}\left(\lambda_{k_{1}} \cdots \lambda_{k_{n-1}}\right)^{\alpha_{\max }} \lambda_{k_{n}}^{\alpha_{\max }} \geqslant C_{1}\left(\frac{a}{A g}\right)^{\alpha_{\max }} A^{\alpha_{\max }} \geqslant C a^{\alpha_{\max }}
\end{aligned}
$$

We are now able to state the main theorem of this section.

Theorem 2. Let $\mu \in \mathscr{M}$. Let $Z_{p, q}(a)$ be its corresponding partition function defined in Eq. (20) and let $D_{q}=\tau(q) /(q-1)$ be its generalized fractal dimensions [Eqs. (15) and (16)]. Then, $\forall q \in \mathbb{R}, \tau(q)$ is the transition exponent so that

$$
\begin{aligned}
& p<\tau(q) \Rightarrow \lim _{a \rightarrow 0^{+}} Z_{p, q}(a)=0 \\
& p>\tau(q) \Rightarrow \lim _{a \rightarrow 0^{+}} Z_{p, q}(a)=+\infty
\end{aligned}
$$

Proof. From Lemma 6 it follows that $\forall \varepsilon \in \mathbb{R}^{+*}$, $\sup _{[\varepsilon, A g]} Z_{p, q}<\infty$ (for negative $q$ ) and that $\inf _{[\varepsilon, A g]} Z_{p, q}>0$ (for positive $q$ ). Moreover, since $\int d \mu(x)=1$, then $\forall \varepsilon \in \mathbb{R}^{+*}$, inf $[\varepsilon, \Delta g] Z_{p, q}>0$ (for negative $q$ ) and $\sup _{[\varepsilon, A g]} Z_{p, q}<\infty$ (for positive $q$ ). We then apply Lemma 3 to $f=Z_{p, q}$.

\section{DETERMINING THE SINGULARITY SPECTRUM OF A FUNCTION FROM ITS WAVELET TRANSFORM MAXIMA LINES}

The purpose of this section is to present a method ${ }^{(31,32)}$ to determine the singularity spectrum (see Section 4.1 ) of a function $f$ which displays a fractal recursive singular structure. We will consider the class of functions $f$ corresponding to the distribution functions of the measures $\mu$ in $\mathscr{M}$ "perturbed" by a $C^{\infty}$ function $r$, i.e.,

$$
f(x)=\int_{0}^{x} d \mu+r(x)
$$

In the following sections, we will study the case where $r$ is a polynomial and then generalize it to any $C^{\infty}$ function. Let us first define what we call the singularity spectrum of a function. 


\subsection{Singularity Spectrum of a Function}

The singularity spectrum $f(\alpha)$ of a multifractal measure $\mu$ is defined as the Hausdorff dimension of the set ${ }^{(2)}$

$$
S_{\alpha}=\left\{x_{0} \in \operatorname{Supp} \mu / \alpha\left(x_{0}\right)=\alpha\right\}
$$

where $\alpha\left(x_{0}\right)$ describes the local behavior of $\mu$ around $x_{0}$, i.e., it is the largest exponent $\alpha$ so that

$$
\int_{\left|x-x_{0}\right|<\varepsilon} d \mu(x)=o\left(\varepsilon^{\alpha}\right) \quad \text { for } \quad \varepsilon \rightarrow 0^{+}
$$

We will define, in the same way, the singularity spectrum $D(h)$ of a function $f$ from its Hölder exponents. ${ }^{(45,46)}$

In the following, $f(\alpha)$ will always denote a singularity spectrum of a function, and $f(x)$ will denote the function which is analyzed.

Definition 3. A function $f$ is said to be of Hölder exponent $h\left(x_{0}\right)$, at the point $x_{0} \in \mathbb{R}$, iff $h\left(x_{0}\right)$ is the largest exponent $h$ such that there exists a constant $A \in \mathbb{R}^{+}$and a polynomial $P_{n}(x)$ of order $n$ such that for all $x$ in a neighborhood of $x_{0}$

$$
\left|f(x)-P_{n}\left(x-x_{0}\right)\right| \leqslant A\left|x-x_{0}\right|^{h}
$$

If $f$ is $C^{\infty}$, then $h\left(x_{0}\right)=+\infty$ for all $x_{0}$ in $\mathbb{R}$. In the following, $H_{f}$ will denote the set of finite Hölder exponents of $f$. We then naturally define $D(h)$ as follows. ${ }^{(26)}$

Definition 4. The singularity spectrum of a function $f$ is the function $D(h)\left(h \in H_{f}\right)$ such that

$$
D(h)=\operatorname{dim}_{H}\left\{x_{0} \in \mathbb{R} / h\left(x_{0}\right)=h\right\}
$$

where $\operatorname{dim}_{H}$ denotes the Hausdorff dimension. ${ }^{(54)}$

It immediately follows that if $\mu \in \mathscr{M}$, the singularity spectrum $D(h)$ of the function

$$
f(x)=\int_{0}^{x} d \mu+r(x)
$$

[where $r(x)$ is $C^{\infty}$ ] is the singularity spectrum $f(\alpha)$ of the measure $\mu$ :

$$
h=\alpha \Rightarrow D(h)=f(\alpha)
$$


Therefore, extracting $D(h)$ is equivalent to extracting the function $\tau(q)$ corresponding to the measure $\mu[f(\alpha)$ is deduced by Legendre transforming $\tau(q)$; cf. Eq. (17)]. Thus, for the particular case where $r=0$, by combining Theorem 2 (using $\psi^{\prime}$ as the analyzing wavelet) and Eq. (7), one can easily derive a way of determining $\tau(q)$ from the wavelet transform maxima lines of $f$. Let us state the corresponding theorem for the more general case where $r$ is a polynomial.

\subsection{Determining the Singularity Spectrum of $f(x)=\int_{0}^{x} d \mu+P_{n}(x)$ (Where $P_{n}$ Is a Polynomial)}

Theorem 3. Let $\mu \in \mathscr{M}$. Let $P_{n}(x)$ be a polynomial of order $n$ and $f(x)=\int_{0}^{x} d \mu+P_{n}(x)$. Let $\psi$ be an analyzing wavelet with $N>n$ vanishing moments, i.e., $\forall k, 0 \leqslant k \leqslant n, \int x^{k} \psi(x) d x=0$. Let $Z_{p, q}^{f}$ be the corresponding partition function [Eq. (20)]:

$$
Z_{p, q}^{f}(a)=a^{-p} \sum_{l \in \mathscr{L}(a)}\left(\sup _{\left(x, a^{\prime}\right) \in l}\left|T_{\psi}[f]\left(x, a^{\prime}\right)\right|\right)^{q}
$$

Then, for all $q$ in $\mathbb{R}, \tau(q)$ [Eq. (16)] is the transition exponent such that

$$
\begin{aligned}
& p<\tau(q) \Rightarrow \lim _{a \rightarrow 0^{+}} Z_{p, q}^{f}(a)=0 \\
& p>\tau(q) \Rightarrow \lim _{a \rightarrow 0^{+}} Z_{p, q}^{f}(a)=+\infty
\end{aligned}
$$

The singularity spectrum of $f$ is then obtained by Legendre transforming $\tau(q)$.

Proof. According to the definition of the wavelet transform of a function $f$ [Eq. (5)],

$$
\begin{aligned}
T_{\psi}[f](b, a) & =\frac{1}{a} \int \psi\left(\frac{x-b}{a}\right) f(x) d x \\
& =T_{\psi}\left[\int_{0}^{x} d \mu\right](b, a)+\frac{1}{a} \int \psi\left(\frac{x-b}{a}\right) P_{n}(x) d x
\end{aligned}
$$

Using Eq. (7), we obtain

$$
T_{\psi}[f](b, a)=-T_{\Psi}[\mu](b, a)+\frac{1}{a} \int \psi\left(\frac{x-b}{a}\right) P_{n}(x) d x
$$


where $\Psi$ denotes the following primitive of $\psi: \Psi(x)=\int_{-\infty}^{x} \psi(t) d t$. Moreover, since $\psi$ has $N$ vanishing moments with $N>n$, we have

$$
\frac{1}{a} \int \psi\left(\frac{x-b}{a}\right) P_{n}(x) d x=\int \psi(x) P_{n}(b+a x) d x=0
$$

and, therefore,

$$
T_{\psi}[f](b, a)=-T_{\Psi}[\mu](b, a)
$$

Then, by applying Theorem 2 (with the analyzing wavelet $\Psi$ ), it follows that $\tau(q)$ is the transition exponent corresponding to $Z_{p, q}^{f}$.

Remark. It is very important to notice that the proof of Theorem 3 required the use of Theorem 2 with the analyzing wavelet $\Psi(x)=$ $\int_{-\infty}^{x} \psi(t) d t$, which has $N-1=n$ vanishing moments ( $\Psi$ is thus oscillating around 0 ). Such an analyzing wavelet makes the control of the part where $T_{\Psi}$ is close to 0 very difficult and therefore keeps us from defining the partition function as an overall sum of the wavelet coefficients raised to the $q$ power [as in Eq. (18)]. Using a partition function based on the wavelet transform modulus maxima seems to be the most "natural" way to circumvent this difficulty. ${ }^{(31-33)}$

Let us now study the general case where $r$ is any $C^{\infty}$ function.

\subsection{Determining the Singularity Spectrum of $f(x)=\int_{0}^{x} d \mu+r(x)$ (Where $r$ Is $C^{\infty}$ )}

Let $s(x)=\int_{0}^{x} d \mu$ and let $\mathscr{L}_{f}$ and $\mathscr{L}_{s}$ be the sets of the maxima lines of the wavelet transform of $f$ and of $s$, respectively. In this section, we will suppose that $\psi$ satisfies:

(H5) $\forall k, 0 \leqslant k<N, \int x^{k} \psi(x) d x=0$ and $\int x^{N} \psi(x) d x \neq 0$.

Let us prove that the wavelet transform of $r$ is of the order of $a^{N}$ when $a$ goes to zero. ${ }^{(40)}$

Lemma 7. There exists a $C^{\infty}$ function $R(b, a)$ uniformly bounded for $a \in\left[0, a_{0}\right]$ (and $b$ in a compact) such that

$$
T_{\psi}[r](b, a)=a^{N} R(b, a)
$$

Proof. By definition of the wavelet transform,

$$
T_{\psi}[r](b, a)=\frac{1}{a} \int \psi\left(\frac{x-b}{a}\right) r(x) d x=\int \psi(x) r(b+a x) d x
$$


Then, by replacing $r(b+a x)$ by its Taylor expansion around $b$, we get

$$
T_{\psi}[r](b, a)=\sum_{k=0}^{N-1} \int \psi(x) \frac{(a x)^{k}}{k !} r^{(k)}(b)+\int \psi(x)(a x)^{N} \varepsilon(b, a x) d x
$$

where $\varepsilon$ is a $C^{\infty}$ function uniformly bounded for $a \in\left[0, a_{0}\right]$ (and $b$ in a compact). The first $N$ terms of the right-hand side of this equation vanish [cf. (H5)]; it follows that

$$
T_{\psi}[r](b, a)=a^{N} R(b, a)
$$

where $R$ is $C^{\infty}$ and uniformly bounded for $a \in\left[0, a_{0}\right]$ (and $b$ in a compact).

We then obtain, from the definition of $f, T_{\psi}[f](b, a)=$ $T_{\psi}[s](b, a)+a^{N} R(b, a)$. This means that we have "perturbed" the wavelet transform of $s$, at scale $a$, by a term of the order of $a^{N}$. The purpose of this section is to study how this term perturbs the behavior of the partition function $Z_{p, q}^{f}$ [Eq. (30)] at small scales, i.e., to compare the limit when $a$ goes to 0 of the two following functions:

$$
Z_{p, q}^{s}(a)=a^{-p} \sum_{l \in \mathscr{L}_{s}(a)}\left(\sup _{\left(x, a^{\prime}\right) \in l}\left|T_{\psi}[s]\left(x, a^{\prime}\right)\right|\right)^{q}
$$

and

$$
Z_{p, q}^{f}(a)=a^{-p} \sum_{l \in \mathscr{L}_{f}(a)}\left(\sup _{\left(x, a^{\prime}\right) \in l}\left|T_{\psi}[f]\left(x, a^{\prime}\right)\right|\right)^{q}
$$

For that purpose we need to study the structure of $\mathscr{L}_{f}$ with the specific goal to relate it to the structure of $\mathscr{L}_{s}$.

Lemma 8. Let $l \in \mathscr{L}_{f}$ and let $b(t)$ and $\left.\left.a(t) \quad(t \in] 0,1\right]\right)$ be two continuous functions such that $\{(b(t), a(t))\}_{t \in j 0,1]} \subset l$ and $\lim _{t \rightarrow 0} a(t)=0$. Then, $\exists b_{l} \in \mathbb{R}$ such that $\lim _{t \rightarrow 0} b(t)=b_{l}$.

Proof. The proof of this lemma is given in Appendix B.

Let us first examine the case where $b_{l}$ does not belong to $J$ (the support of $\mu$ ); since $J$ is a closed set, there exists $\varepsilon>0$ such that ]$b_{l}-\varepsilon, b_{l}+\varepsilon[\cap J=\phi$ and thus [using (H1)], for $t$ small enough, $T_{\psi}[s](b(t), a(t))=0$. Therefore, $T_{\psi}[f](b(t), a(t))=a(t)^{N} R(b(t), a(t))$ and

$$
\sup _{a(t) \leqslant a}\left|T_{\psi}[f](b(t), a(t))\right|=O\left(a^{N}\right)
$$

The maxima line represented by $(b(t), a(t))$ is a maxima line of $T_{\psi}[r]$. 
What about the maxima lines of $T_{\psi}[s]$ ? How are they perturbed? The following lemma partially answers this question.

Lemma 9. Let us suppose that:

(H6) $N>\max \left(\alpha_{\max }, 3 \alpha_{\max } / 2-1\right)$ [see (H5) for the definition of $N$ and Lemma 6 for the definition of $\alpha_{\max }$ ].

(H7) $d\left(\partial T_{\psi}[s] / \partial b\right)=\left(\partial^{2} T_{\psi}[s] / \partial b^{2}\right) d b+\left(\partial^{2} T_{\psi}[s] / \partial a \partial b\right) d a \neq 0$ on any maxima line in $\mathscr{L}_{s}$.

(H8) $\partial^{2} T_{\psi}[s] / \partial b^{2} \neq 0$ on any maxima line in $\mathscr{L}_{s}$, except at its boundary points.

Then for all $\varepsilon>0$, there exists $a_{0}>0, k>1$, and $C>0$ such that

$\forall a<a_{0}, \quad \forall l \in \mathscr{L}_{s}(a), \quad a_{l}>a /(1-\varepsilon) \quad$ then $\quad \exists ! l_{1} \in \mathscr{L}_{f}(a)$ such that

$$
\forall\left(x, a^{\prime}\right) \in l, \quad \exists !\left(x_{1}, a^{\prime}\right) \in l_{1}, \quad\left|x_{1}-x\right|<C a^{\prime k}
$$

where $a_{l}$ is defined as in Notation 3 (in Section 2). Moreover,

$$
T_{\psi}[f]\left(x_{1}, a^{\prime}\right)=T_{\psi}[s]\left(x, a^{\prime}\right)\left[1+\eta\left(x, a^{\prime}\right)\right]
$$

where $\eta\left(x, a^{\prime}\right)$ is a function which is uniformly bounded by a decreasing function $F\left(a^{\prime}\right)$ (independent of $l$ ) which goes to 0 when $a^{\prime}$ goes to 0 .

In the following, $P(l)$ will denote the corresponding perturbed line $l_{1}$.

Proof. The proof is given in Appendix C.

This lemma mainly says that if we consider a maxima line $l \in \mathscr{L}_{s}(a)$ and if we are not too close to the point where this line just "appears" [i.e., $\left.a_{l}>a /(1-\varepsilon)\right]$, then $l$ is just shifted when perturbing $s$ by $r$. Moreover, the value of $T_{\psi}$ on this new maxima line is slightly changed. However, this lemma does not say anything about what happens near the point where the line appears (i.e., $a$ close to $a_{l}$ ). If $l \in \mathscr{L}_{s}(a), a_{l}<\infty$, then it is easy to prove that $a_{l}$ corresponds to an abscissa $x_{l}$ so that $\left(x_{l}, a_{l}\right)$ is the point of the $(b, a)$ half-plane where $l$ "appears." From (H7) it follows that this point can be only of two types:

1. The value of $T_{\psi}[s]$ at this point is 0 .

2. The value of $\partial^{2} T_{\psi}[s] / \partial b^{2}$ at this point is 0 and $\partial^{2} T_{\psi}[s] / \partial a \partial b \neq 0$. For each case we want to find a way to control $a_{P(l)}$ with respect to $a_{l}$.

Lemma 10. Let us suppose that:

(H9) If both $T_{\psi}[s]$ and $\partial T_{\psi}[s] / \partial b$ are vanishing at a certain point, then both $\partial T_{\psi}[s] / \partial a$ and $\partial^{2} T_{\psi}[s] / \partial b^{2}$ are nonzero. 
Then $\exists \varepsilon, \exists a_{0}, \forall l \in \mathscr{L}_{s}(a), a_{l}>a /(1-\varepsilon)$,

$$
\text { if } a_{0}>a_{l}, \quad \text { then } a_{P(l)}<a_{l} /(1-\varepsilon)
$$

Proof. The proof is given in Appendix D.

From Lemmas 9 and 10 , it follows that a line $l_{1} \in \mathscr{L}_{f}\left(a_{1}\right)$ "comes" from the perturbation of a line in $\mathscr{L}_{s}$ if and only if $\exists l \in \mathscr{L}_{s}(a)$, $(1-\varepsilon) a<a_{1}<a /(1-\varepsilon)$ such that $P\left(\left.l\right|_{a(1-\varepsilon)}\right)=\left.l_{1}\right|_{a(1-\varepsilon)}$. This will be used later for the definition of the set $\mathscr{L}_{P}(a)$.

Then the "end" of a line $\left(x_{l}, a_{l}\right)$, when perturbated, can move at most by a factor of $(1-\varepsilon)$ toward small scales or large scales. We now need to find a way to control the value of $T_{\psi}[f]$ around the end of the line. It can be proved easily that $\forall \varepsilon, \exists C>0$ such that

$$
\forall l \in \mathscr{L}_{s}(a) \quad \sup _{l}\left|T_{\psi}[s]\right|<C \sup _{l \mid(1-\varepsilon)^{2} a}\left|T_{\psi}[s]\right|
$$

Indeed, we first compare

$$
S_{1}=\sup _{l}\left|T_{\psi}[s]\right| \quad \text { and } \quad S_{2}=\sup _{l \mid(1-\varepsilon)^{2} a}\left|T_{\psi}[s]\right|
$$

for all $a$ in $[A g, g]$ and for all the maxima lines $l$ in $\mathscr{L}_{s}(a)$; we so obtain a constant $C$ such that (37) holds; we then use Lemma 5 to extend it to all scales $a>0$. From Lemma 9 it follows that the relation (37) holds when replacing $l$ by $l_{1}=P(l)$. By considering the same two cases as in Appendix D, one can easily prove that it extends to any line which "comes" from the perturbation of a line in $\mathscr{L}_{s}$, i.e., we have the following result:

Lemma 11. There exists $C(>1)$ such that for any line $l_{1} \in \mathscr{L}_{f}\left(a_{1}\right)$ which comes from the perturbation of the line $l \in \mathscr{L}_{s}(a)$ [i.e., $(1-\varepsilon) a<$ $a_{1}<a /(1-\varepsilon)$ such that $\left.P\left(\left.l\right|_{a(1-\varepsilon)}\right)=\left.l_{1}\right|_{a(1-\varepsilon)}\right]$ we have

$$
\sup _{l_{1}}\left|T_{\psi}[f]\right|<C \sup _{l_{1} \mid(1-\varepsilon)^{2} a_{1}}\left|T_{\psi}[f]\right|
$$

Up to now, we have found two kinds of maxima lines in $\mathscr{L}_{f}$, those which were maxima lines of $T_{\psi}[r]$ [Eq. (34)] and those which came from the perturbation of the maxima lines in $\mathscr{L}_{s}$. Let us suppose that they are the only ones, i.e., $\mathscr{L}_{f}\left(a_{1}\right)$ can be subdecomposed (for $a_{1}<a_{0}$ ) into two subsets: the set $\mathscr{L}_{P}\left(a_{1}\right)$ and the set $\mathscr{L}_{N}\left(a_{1}\right)$ :

$$
\mathscr{L}_{f}\left(a_{1}\right)=\mathscr{L}_{P}\left(a_{1}\right) \oplus \mathscr{L}_{N}\left(a_{1}\right)
$$


where

$$
\begin{aligned}
\mathscr{L}_{P}\left(a_{1}\right)= & \left\{l_{1} \in \mathscr{L}_{f}\left(a_{1}\right) / \exists l \in \mathscr{L}_{s}(a),(1-\varepsilon) a<a_{1}<a /(1-\varepsilon),\right. \\
& \left.\left.P\left(\left.l\right|_{a(1-\varepsilon)}\right) \subset l_{1}\right|_{a(1-\varepsilon)}\right\} \\
\mathscr{L}_{N}\left(a_{1}\right)= & \left\{l \in \mathscr{L}_{f}\left(a_{1}\right) / \sup \left|T_{\psi}\right| \sim a^{\prime N} \text { when } a^{\prime} \rightarrow 0\right\}
\end{aligned}
$$

We will also suppose that for small enough $a, \mathscr{L}_{N}(a)$ has a finite constant number of elements $n_{N} \neq 0$. We can then compare the two partition functions $Z_{p, q}^{s}$ [Eq. (32)] and $Z_{p, q}^{f}$ [Eq. (33)].

Lemma 12. $\forall q \in \mathbb{R}, \exists\left(C_{1}, C_{2}\right) \in \mathbb{R}^{+^{2}}$,

$$
\begin{aligned}
Z_{p, q}^{f}(a(1-\varepsilon)) & \geqslant C_{1}\left(a^{N q-p}+Z_{p, q}^{s}(a)[1+o(1)]\right) \\
Z_{p, q}^{f}(a /(1-\varepsilon)) & \leqslant C_{2}\left(a^{N q-p}+Z_{p, q}^{s}(a)[1+o(1)]\right)
\end{aligned}
$$

when $a$ goes to 0 ( $\varepsilon$ is defined in Lemma 10 ).

Proof. We will only give the proof of the second inequality; the first one can be proved in the same way. From the definition (33), it follows that (we use the variable name $a_{1}$ instead of $a$ to be consistent with the notations of Lemmas 9 and 10)

$$
\begin{aligned}
Z_{p, q}^{f}\left(\frac{a_{1}}{1-\varepsilon}\right)= & \frac{(1-\varepsilon)^{p}}{a_{1}^{p}} \sum_{\left.l_{1} \in \mathscr{L}_{f}\left(a_{1} /(1-\varepsilon)\right)\right)}\left(\sup _{l_{1}}\left|T_{\psi}[f]\right|\right)^{q} \\
= & \frac{(1-\varepsilon)^{p}}{a_{1}^{p}}\left(\sum_{l_{1} \in \mathscr{L}_{P}\left(a_{1} /(1-\varepsilon)\right)}\left(\sup _{l_{1}}\left|T_{\psi}[f]\right|\right)^{q}\right. \\
& \left.+\sum_{l_{1} \in \mathscr{L}_{N}\left(a_{1} /(1-\varepsilon)\right)}\left(\sup _{l_{1}}\left|T_{\psi}[f]\right|\right)^{q}\right)
\end{aligned}
$$

By definition of $\mathscr{L}_{N}$, the second term is

$$
\sum_{l_{1} \in \mathscr{L}_{N}\left(a_{\ell} /(1-\varepsilon)\right)}\left(\sup _{l_{1}}\left|T_{\psi}[f]\right|\right)^{q}<C n_{N} a_{1}^{N q}
$$

On the other hand, we get [Eq. (38) is used for positive values of $q$ ]

$$
\begin{aligned}
& \sum_{l_{1} \in \mathscr{L}_{P\left(a_{1} /(1-\varepsilon)\right)}}\left(\sup _{l_{1}}\left|T_{\psi}[f]\right|\right)^{q}=\sum_{l_{1} \in \mathscr{L}_{P\left(a_{1} /(1-\varepsilon)\right)}}\left(\sup _{l_{1} \mid a_{1} /(1-\varepsilon)}\left|T_{\psi}[f]\right|\right)^{q} \\
& \leqslant C^{\prime} \sum_{l_{1} \in \mathscr{L}_{P}\left(a_{1} /(1-\varepsilon)\right)}\left(\sup _{l_{1} \mid a_{1}(1-\varepsilon)}\left|T_{\psi}[f]\right|\right)^{q}
\end{aligned}
$$


Moreover, if $l_{1} \in \mathscr{L}_{P}\left(a_{1} /(1-\varepsilon)\right)$, then $l_{1}$ "comes" from a perturbed line $l \in \mathscr{L}_{s}(a)$, i.e. (cf. definition of $\left.\mathscr{L}_{P}\right)$, with $a>a_{1}>(1-\varepsilon)^{2} a$ and $P\left(\left.l\right|_{a(1-\varepsilon)}\right)=$ $\left.l_{1}\right|_{a(1-\varepsilon)}$. As $a(1-\varepsilon)>a_{1}(1-\varepsilon)$, we also have $P\left(\left.l\right|_{a_{1}(1-\varepsilon)}\right)=\left.l_{1}\right|_{a_{1}(1-\varepsilon)}$. It thus follows that

$$
\sum_{l_{1} \in \mathscr{L}_{P\left(a_{1} /(1-\varepsilon)\right)}}\left(\sup _{l_{1}}\left|T_{\psi}[f]\right|\right)^{q} \leqslant C^{\prime} \sum_{l \in \mathscr{\mathscr { L }}_{s}(a)}\left(\sup _{P\left(\left.l\right|_{\left.a_{1}(1-\varepsilon)\right)}\right.}\left|T_{\psi}[f]\right|\right)^{q}
$$

By using Lemma 9, we obtain

$$
\begin{aligned}
\sum_{l_{1} \in \mathscr{P}_{P}\left(a_{1} /(1-\varepsilon)\right)}\left(\sup _{l_{1}}\left|T_{\psi}[f]\right|\right)^{q} & \leqslant C^{\prime} \sum_{l \in \mathscr{L}_{s}(a)}\left(\sup _{\left.l\right|_{a_{1}(1-\varepsilon)}}\left|T_{\psi}[s](1+\eta)\right|\right)^{q} \\
& \leqslant C^{\prime \prime} \sum_{l \in \mathscr{L}_{s}\left(a_{1}\right)}\left(\sup _{l_{a_{1}}}\left|T_{\psi}[s](1+\eta)\right|\right)^{q}
\end{aligned}
$$

where we have used Eq. (37) for $q<0$. Note that from Lemma 9, $\eta\left(x, a^{\prime}\right)$ is a function which is uniformly (in $x$ ) bounded by a decreasing function $F\left(a^{\prime}\right)$ independent of $l$ which goes to 0 when $a^{\prime}$ goes to 0 . Then we get

$$
\begin{aligned}
\sum_{l_{1} \in \mathscr{L}_{P}\left(a_{1} /(1-\varepsilon)\right)}\left(\sup _{l_{1}}\left|T_{\psi}\right|\right)^{q} & \leqslant C^{\prime \prime \prime} \sum_{l \in \mathscr{\mathscr { S }}_{s}\left(a_{1}\right)}\left(\sup _{l}\left|T_{\psi}[s]\right|\right)^{q}\left[1+F\left(a_{1}\right)\right]^{q} \\
& \leqslant C^{\prime \prime \prime} \sum_{l \in \mathscr{S}_{s}\left(a_{1}\right)}\left(\sup _{l}\left|T_{\psi}[s]\right|\right)^{q}[1+o(1)]
\end{aligned}
$$

By inserting (41) and (42) in (40), we finally obtain

$$
Z_{p, q}^{f}\left(a_{1} /(1-\varepsilon)\right)<C_{1}\left(n_{N} a_{1}^{N q-p}+Z_{p, q}^{s}\left(a_{1}\right)[1+o(1)]\right)
$$

The lower bound for $Z_{p, q}^{f}(a(1-\varepsilon))$ can be computed using the same technique.

We are now ready to state the main theorem of this section.

Theorem 4. There exists $q_{\text {erit }}<0$ such that:

(a) If $q>q_{\text {crit }}$, then $\tau(q)$ is the transition exponent such that

$$
\begin{aligned}
& p<\tau(q) \Rightarrow \lim _{a \rightarrow 0^{+}} Z_{p, q}^{f}(a)=0 \\
& p>\tau(q) \Rightarrow \lim _{a \rightarrow 0^{+}} Z_{p, q}^{f}(a)=+\infty
\end{aligned}
$$

(b) If $q<q_{\text {crit }}$, then

$$
\begin{aligned}
& p<q N \Rightarrow \lim _{a \rightarrow 0^{+}} Z_{p, q}^{f}(a)=0 \\
& p>q N \Rightarrow \lim _{a \rightarrow 0^{+}} Z_{p, q}^{f}(a)=+\infty
\end{aligned}
$$


Proof. Let us first recall that $\tau(q)$ is an increasing convex function ${ }^{(2,9,10)}$ that satisfies

$$
\begin{aligned}
& \tau(q) \sim(q-1) \alpha_{\max } \quad \text { when } \quad q \rightarrow-\infty \\
& \tau(0)<0
\end{aligned}
$$

Then, as $N>\alpha_{\max }$ [cf. (H6)], there exists a unique $q=q_{\text {crit }}<0$ such that $\tau(q)=N q$. Moreover, as $\tau(q)$ is convex:

(a) $q>q_{\text {crit }} \Rightarrow \tau(q)<N q$.

(b) $q<q_{\text {crit }} \Rightarrow \tau(q)>N q$.

Let us distinguish the two cases:

(a) $q>q_{\text {crit }}[N q>\tau(q)]$. We distinguish two situations:

(i) $p<\tau(q)$. By using Theorem 3 (with $r=0$ ) we get $\lim Z_{p, q}^{s}(a)=0$. Moreover, $N q-p>0$ and from Lemma 12 one deduces $\lim Z_{p, q}^{f}(a)=0$.

(ii) $p>\tau(q)$. By using Theorem 3 (with $r=0$ ) we get $\lim Z_{p, q}^{s}(a)=$ $+\infty$, and thus from Lemma $12, \lim _{a \rightarrow 0} Z_{p, q}^{f}(a)=+\infty$.

(b) $q<q_{\text {crit }}[N q<\tau(q)]$. We distinguish again two situations:

(i) $p>N q$. Then $N q-p<0$, and therefore $a^{N q-p} \rightarrow \infty$. From the lower bound of Lemma 12, one gets $\lim Z_{p, q}^{f}(a)=\infty$.

(ii) $p<N q<\tau(q)$. As $p<\tau(q)$, one gets from Theorem $3(r=0)$ that $\lim Z_{p, q}^{s}(a)=0$. Moreover, $a^{N q-p} \rightarrow 0$, and from the upper bound of Lemma 12 it follows that $\lim Z_{p, q}^{f}(a)=0$.

Let us comment on this theorem before moving on to numerical applications.

\subsection{Some Important Comments on Theorem 4}

Phase Transition Phenomenon. From Theorem 4, we conclude that in the case where some maxima lines of $\mathscr{L}_{f}$ decrease like $a^{N}$ (i.e., some maxima lines of $T_{\psi}[r]$ are converging, in the sense of Lemma 8, toward $\left.b_{l} \notin J\right)$, then we are not able to recover the whole function $\tau(q)$. Indeed, we can extract numerically (by studying the transition exponent of $Z_{p, q}^{f}$ ) a function $\tau_{N}(q)$ that matches $\tau(q)$ only for $q>q_{\text {erit }}\left(q_{\text {crit }}<0\right)$ :

$$
\begin{aligned}
& q>q_{\text {crit }} \Rightarrow \tau_{N}(q)=\tau(q) \\
& q<q_{\text {crit }} \Rightarrow \tau_{N}(q)=N q
\end{aligned}
$$

This nonanalyticity of the function $\tau(q)$ expresses the breaking of the self-similarity of the underlying singular measure by the $C^{\infty}$ perturbation 
$r(x)$. In the context of a thermodynamic analogy, this phenomenon corresponds to a phase transition. ${ }^{(12,48-51)}$ Below the critical value $q_{\text {crit }}$ (which corresponds to the transition temperature) one observes a regular phase, whereas for $q>q_{\text {crit }}$ one switches to a singular (multifractal) phase. Then, if $D(h)$ denotes the singularity spectrum of $f$ and $D_{N}(h)$ the one we deduce from $\tau_{N}(q)$ [by Legendre transforming $\tau_{N}(q)$, Eq. (17)], the curve $y=D_{N}(h)$ can be seen as the curve $y=D(h)$ in which a part is replaced by the line which is tangent to the curve and which passes by the point $(h=N$, $y=0$ ) (see Fig. 2d). Of course, if $N$ is changed, then the shape of $D_{N}(h)$ changes. Conversely, if, when we change $N$, the singularity spectrum we measure $D_{N}(h)$ does not change, then it is likely that $T_{\psi}[r]$ does not "interfere" in the measurement of $D(h)$. Indeed, if it was interfering, it would mean that there would be some maxima lines in $\mathscr{L}_{f}$ along which the decay of $T_{\psi}[f]$ would depend in a certain way on $T_{\psi}[r]$. As $T_{\psi}[r]$ directly depends upon the order $N$ of the analyzing wavelet $\psi$ (cf. Lemma 7 ), we would expect a change in $D_{N}(h)$ when changing $N$ (i.e., changing the analyzing wavelet).

Consequently, if we use the technique corresponding to Theorem 4 to measure the singularity spectrum of an experimental signal $u(x)$ [i.e., measuring numerically the transition exponents of the partition function $Z_{p, q}^{u}$ as defined in Eq. (30)], then a good test to find out whether our measurements corresponding to $q<0$ are "reliable" or not consists in proceeding to different measurements of $D(h)$ using different analyzing wavelets $^{(31-33)}$ (e.g., $\left.\psi, \psi^{\prime}, \psi^{\prime \prime}, \ldots\right)$ in order to check whether or not the $D(h)$ curve is sensitive to the shape of the analyzing wavelet.

Measuring the Hausdorff Dimension of the Set Which Supports the Singularities of $f$. If $f$ is a function, one can prove that the maximum value of $D(h)$ corresponds to the Hausdorff dimension of the set of the abscissa where $f$ is singular [i.e., $\operatorname{dim}_{H}\{x \in \mathbb{R}, h(x) \neq+\infty\}$ ]. Note that from the Legendre transform, the maximum value is obtained for $q=0$. Let us point out that, even in the presence of a $C^{\infty}$-behavior-induced phase transition, this Hausdorff dimension is never alterated provided $q_{\text {crit }}<0$.

\section{NUMERICAL APPLICATIONS}

In this section, we report some numerical applications that illustrate the relevance and the generality of the theoretical results derived in this paper. Even though we have established our main theorems for analyzing wavelets with compact support, we will carry out our numerical examples with analyzing wavelets that belong to the class of commonly used 
real-valued wavelets defined by the successive derivatives of the Gaussian function ${ }^{(34-38)}$ :

$$
\psi^{(k)}=(-1)^{k+1} \frac{d^{k}}{d x^{k}}\left(e^{-x^{2} / 2}\right)
$$

These wavelets are actually well localized in both direct and Fourier spaces and thus are well adapted to the spectral algorithm we use to compute the continuous wavelet transform. Moreover, the fast decrease of the Gaussian functions when $x$ goes to infinity is sufficient for our theoretical demonstrations to remain valid, as pointed out in Section 2 when stating the working hypotheses $(\mathrm{H} 1)$ and $(\mathrm{H} 2)$.

Example 1. Our first application concerns a signal whose singular part $s(x)$ is the distribution function of a Bernoulli measure $\mu \in \mathscr{M}$ over the unit interval $A=[0,1]$. More precisely, $\mu$ is the invariant measure of one member of the class of expanding piecewise linear maps $T$ defined in Section 3.1, with $s=2, \lambda_{1}=\lambda_{2}=1 / 3, p_{1}=0.6$, and $p_{2}=0.4$. Thus $T^{-1}(A)$ is the union of two disjoint intervals separated by a gap $g=1 / 3$. The so-obtained devil staircase $s(x)$ is actually perturbed by the addition of a $C^{\infty}$ function $r(x)=R \sin (8 \pi x)$, i.e., a sine function over four periods. The signal $f(x)=s(x)+r(x)$ is shown in Fig. 1a. The set of the maxima lines of the
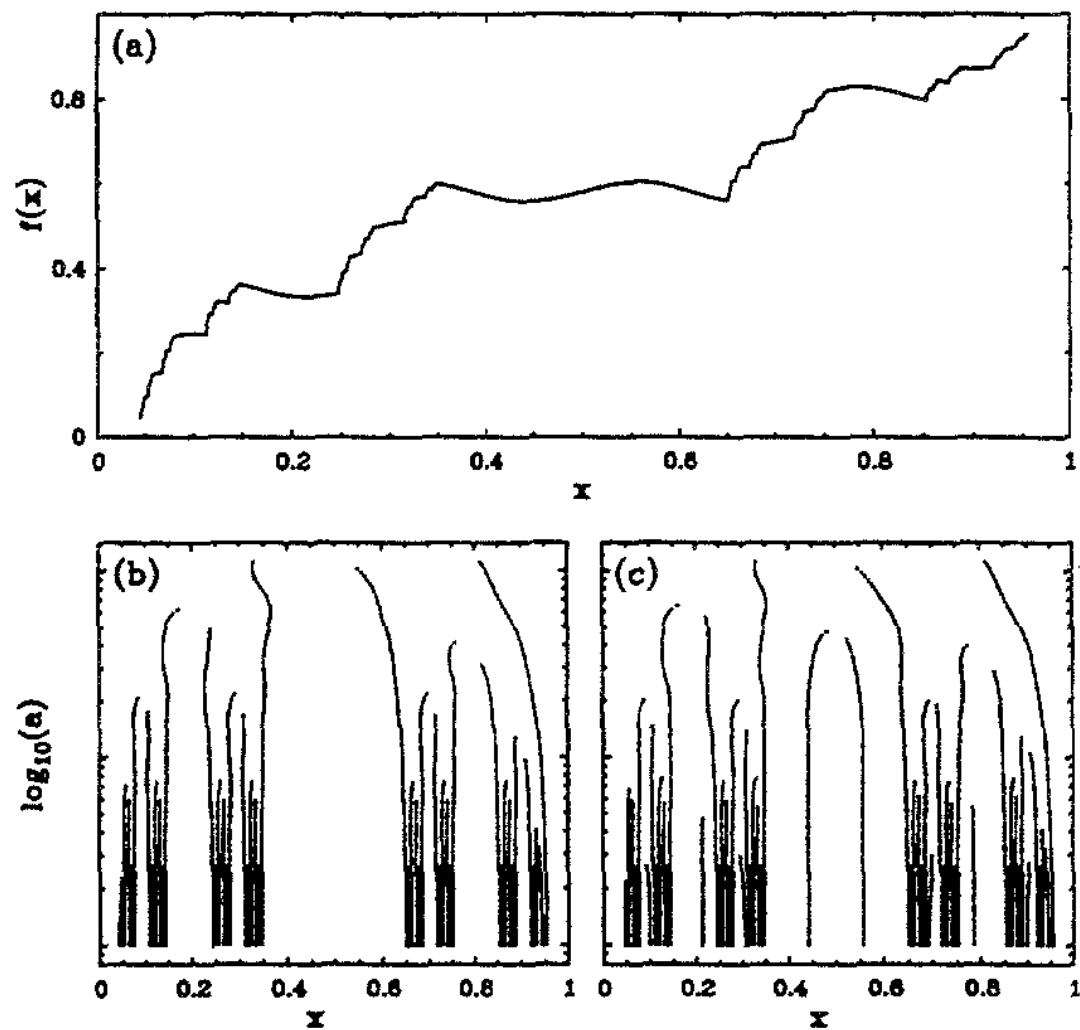

Fig. 1. Maxima lines of the wavelet transform of the function $f(x)=s(x)+r(x)$, where $r(x)=R \sin (8 \pi x)$ and $s(x)$ is the distribution function of the measure $\mu \in \mathscr{M}$ with $s=2$, $\lambda_{1}=\lambda_{2}=1 / 3, p_{1}=0.6$, and $p_{2}=0.4$. (a) Graph of $f(x)$. (b) Wavelet transform maxima lines of $s(x)$. (c) Wavelet transform maxima lines of $f(x)$. The analyzing wavelet is $\psi^{(2)}(x)=\left(1-x^{2}\right) \exp \left(-x^{2} / 2\right)$. 
wavelet transform of $f(x)$, as computed with the analyzing wavelet $\psi^{2}(x)=\left(1-x^{2}\right) e^{-x^{2} / 2}$, is shown in Fig. 1c. Note that $\psi^{(2)}$ has its first two moments vanishing, which implies $N=2$ from the hypothesis (H5) in Section 4.3. The wavelet transform maxima lines of the devil staircase $s(x)$ alone [without the perturbation $r(x)$ ] are shown in Fig. 1b for comparison. The main observation is that each maxima line in Fig. $1 \mathrm{~b}$ is recovered in the wavelet transform representation of the $C^{\infty}$ perturbed devil staircase in Fig. 1c. Even though the corresponding maxima lines in Figs. $1 \mathrm{~b}$ and $1 \mathrm{c}$ may differ by a slight shift at the largest scales, they converge to the same point of the support of $\mu$ when the scale goes to 0 (the amplitude of the shift goes to zero when a goes to zero; see Lemma 9). But some additional maxima lines can be identified in Fig. 1c. Along these "extra" lines, the amplitude of the wavelet transform $\left|T_{\psi^{(2)}}[f]\right|$ is found to decrease like $a^{N}=a^{2}$, as illustrated in Fig. 2a, where $\left|T_{\psi^{(2)}}[f]\right|$ is plotted versus $a$ in a $\log -\log$ representation. This $a^{N}$ power-law behavior is systematically obtained for each of these additional maxima lines in Fig. 1c.
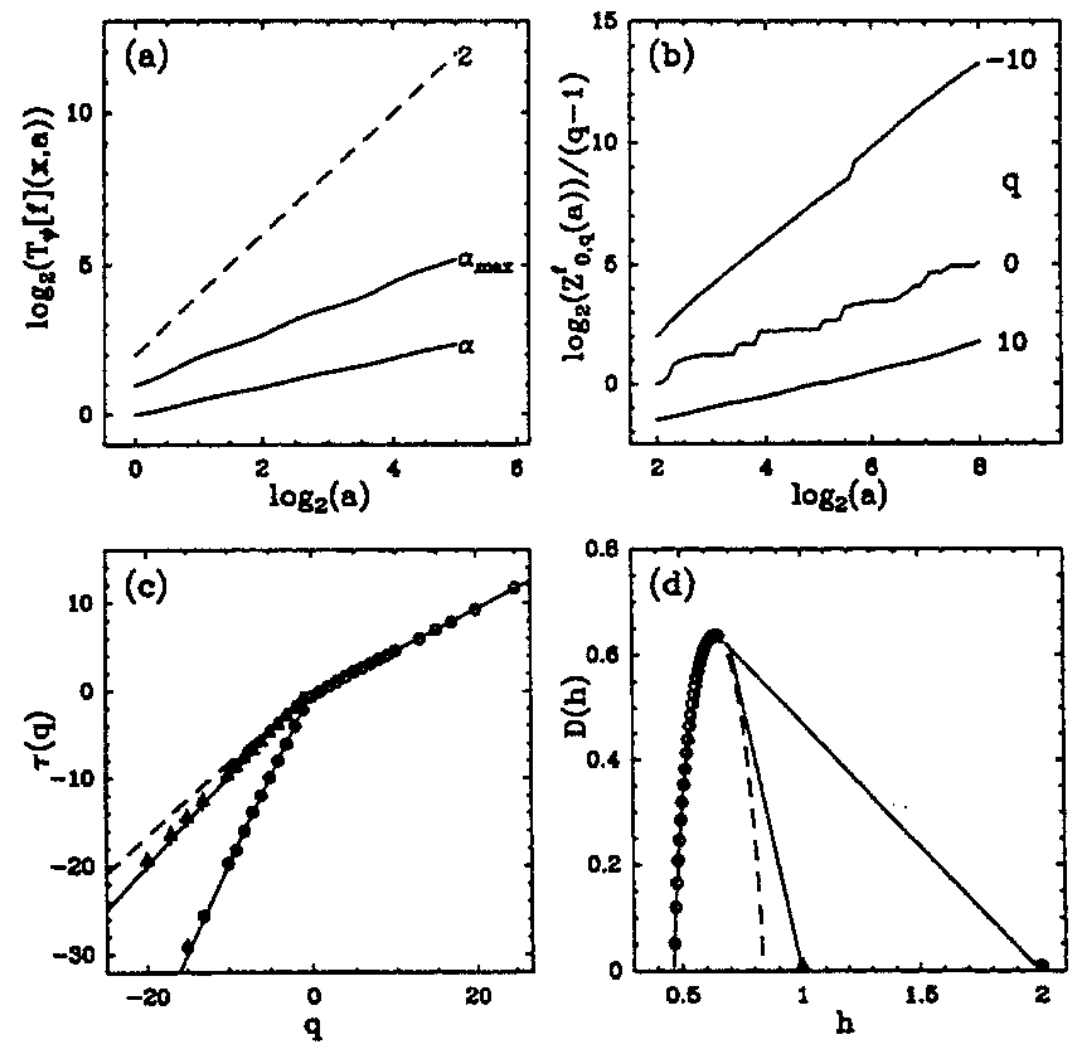

Fig. 2. Measurement of the singularity spectrum of the multifractal signal shown in Fig. 1a. (a) Local scaling exponents revealed in $\log$ - $\log$ plots of $T_{\psi}[f](x, a)$ versus $a$ along maxima lines of $\mathscr{L}_{P}$ (continuous lines) or of $\mathscr{L}_{N}$ (dashed lines). (b) Plot of $\log _{2} Z_{0, q}^{f}(a) /(q-1)$ versus $\log _{2} a$ for different values of $q$. In (a) and (b) the analyzing wavelet is $\psi^{(2)}(x)=\left(1-x^{2}\right) \exp \left(-x^{2} / 2\right)$. (c) $\tau(q)$ versus $q$ as obtained with the analyzing wavelets $\psi^{(1)}(x)=-x \exp \left(-x^{2} / 2\right)(0, \mathbf{\Delta})$ and $\psi^{(2)}(x)(0,0)$; the solid lines correspond to the theoretical predictions from Theorem 4; the dashed line is the part $\left(q<q_{c}\right)$ of the $\tau(q)$ curve of the underlying measure $\mu$, which is masked by the $C^{\infty}$ behavior. (d) $D(h)$ versus $h$ from the Legendre transform of $\tau(q)$; the symbols are the same as in (c). 
It departs significantly from the power-law behavior $\left|T_{\psi^{(2)}}[f]\right| \sim a^{\alpha}$ with $\alpha \leqslant \alpha_{\max }=\ln p_{2} / \ln \hat{\lambda}_{2}=\ln 0.4 / \ln 1 / 3=0.834 \ldots$, extracted along maxima lines emanating from the singular part $s(x)$ of the signal (Fig. 2a). The set $\mathscr{L}_{f}$ of maxima lines of $\left|T_{\psi^{(2)}}[f]\right|$ can thus be decomposed into two subsets: the set $\mathscr{L}_{P}$ of the maxima lines originating from the singularities of $s$, and the set $\mathscr{L}_{N}$ of the maxima lines induced by the $C^{\infty}$ sine contribution. This result can be seen as a numerical illustration of the relevance of Eq. (39), which is the basic hypothesis of our demonstration in Section 4.3. Moreover, the number of extra maxima lines induced by the $C^{\infty}$ behavior is finite and directly related to the number of periods of the sine function.

From the values of $\left|T_{\psi}[f]\right|$ on the set $\mathscr{L}_{f}$ of maxima lines shown in Fig. 1c, one can compute the partition function $Z_{p, q}^{f}(a)$ defined in Eq. (30) and consequently estimate $\tau(q)$ as a transition value for the scaling exponent of $Z_{p, q}^{f}(a)$ in the limit $a \rightarrow 0^{+}$. Practically, $\tau(q)$ is extracted from the scaling behavior of $Z_{0, q}^{f}(a) \sim a^{\tau(q)}$ over a significantly wide range of scales. ${ }^{(31-33)}$ As illustrated in Fig. $2 b$, for three different values of $q=+10,0,-10$, determining $\tau(q)$ just amounts to extracting the slope of $\ln Z_{0, q}^{f}(a)$ versus $\ln a$ from a least-square linear regression fit. The overall results of our $\tau(q)$ measurement are reported in Fig. 2c. The computations have been performed using two different analyzing wavelets: $\psi^{(1)}(x)=-x e^{-x^{2} / 2}(N=1)$ and $\psi^{(2)}(x)=(1-x) e^{-x^{2} / 2}(N=2)$. For $q>0$ (open circles in Figs. $2 \mathrm{c}$ and $2 \mathrm{~d}$ ), both analyzing wavelets lead to numerically identical estimates for $\tau(q)$. On the contrary, for $q<0$, the numerical data obtained with $\psi^{(1)}$ (solid triangles) and $\psi^{(2)}$ (solid circles) separate from each other into two distinct straight lines respectively of slope 1 and 2. We thus observe numerically the phase transition phenomenon predicted by Theorem 4. For positive $q$ values, we recover the $\tau(q)$ spectrum of the underlying singular measure $\mu$ [Eq. (16)], while for $q$ below some critical negative value $q_{\text {crit }}$, the shape of the $\tau(q)$ curve is dictated by the number $N$ of vanishing moments of the analyzing wavelet. Our numerical results in Fig. $2 c$ are in excellent quantitative agreement with the analytical spectra (full lines) predicted by Theorem 4, and clearly deviate from the theoretical $\tau(q)$ spectrum [cf. Eq. (16)] (dashed line) for $q<q_{\text {crit }}$. As far as a precise estimate of the critical value $q_{\text {crit }}$ (which depends on $N$ ) is concerned, large-scale simulations would be necessary to lessen the crossover effect observed around this $q$ value.

By Legendre transforming $\tau(q)$, one gets the $D(h)$ singularity spectrum of $f(x)$. As shown in Fig. 2d, as long as $q>q_{\text {crit }}(N)$, the numerical results obtained with the two analyzing wavelets $\psi^{(1)}$ and $\psi^{(2)}$ (circles) fall remarkably on the theoretical $D(h)$ curve (full line). For $q<q_{\text {crit }}(N)$, however, the Legendre transform of the linear behavior of $\tau(q)$ (cf. Fig. $2 \mathrm{c}$ ) produces a linear falloff of the $D(h)$ curve toward the limiting value $h=1$ 
for $\psi^{(1)}$ and $h=2$ for $\psi^{(2)}$ (actually $h=N$ for $\psi^{(N)}$ ), where $D(h)$ vanishes. This linear part is tangent to the theoretical $D(h)$ spectrum (dashed line) and has a slope equal to $q_{\text {crit }}(N)$. It is the signature of the phase transition phenomenon described in Section 4.4. Thus, in this example, the presence of the $C^{\infty}$ sine behavior prevents us from measuring most of the right-hand side of the $D(h)$ spectrum. But, as pointed out in Section 4.4 , this phenomenon is analyzing wavelet dependent, and the use of different analyzing wavelets provides us with a clear diagnostic of whether or not the weakest singularities are masked by $C^{\infty}$ contributions.

Let us remark, however, that one can practically identify all the maxima lines that belong to the set $\mathscr{L}_{N}$ from the characteristic behavior of the wavelet transform $\left(T_{\psi(N)}[f]_{v} a^{N}\right)$. Then, by computing partition functions restricted to the set $\mathscr{L}_{S}$ one can "restore" the self-similarity and determine the whole singularity spectrum. ${ }^{(33)}$

Example 2. Our second application is an illustration of the robustness of our theoretical results that are likely to extend to a large class of singular functions which do not necessarily meet the conditions required by our mathematical analysis. The signal in Fig. $3 a$ is a random function over $[0,1]$, generated from the distribution function of a measure $\mu$ that does
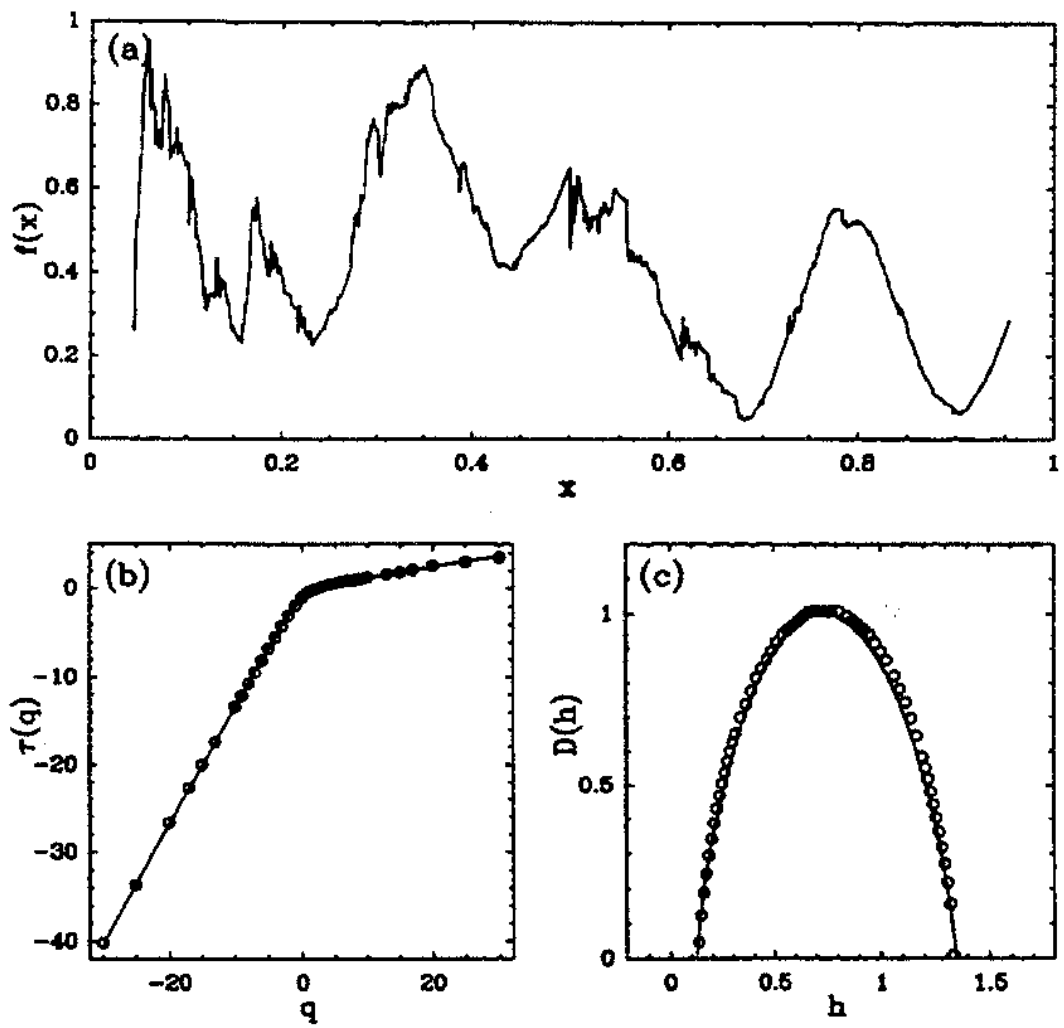

Fig. 3. Measurement of the singularity spectrum of the multifractal signal $f(x)=s(x)+r(x)$, where $r(x)=R \sin (8 \pi x)$ and $s(x)$ is the distribution function of a measure $\mu$ randomly distributed over the whole interval (gap $g=0$ ) with $s=4, \lambda_{1}=\lambda_{2}=\lambda_{3}=\lambda_{4}=1 / 4, p_{1}=0.845$, $p_{2}=-p_{3}=0.362$, and $p_{4}=0.155$ (see text). (a) Graph of a realization of $f$. (b) $\tau(q)$ versus $q$. (c) $D(h)$ versus $h$. In (b) and (c), the solid line corresponds to the analytical spectra. The analyzing wavelet is $\psi^{(2)}(x)=\left(1-x^{2}\right) \exp \left(-x^{2} / 2\right)$. 
not belong to $\mathscr{M}$ since the condition $g>0$ has been relaxed and some of the $p_{i}$ have been allowed to take negative values. More precisely, $\mu$ is constructed along the lines of the definitions in Section 3.1, with $s=4$, $\lambda_{1}=\lambda_{2}=\lambda_{3}=\lambda_{4}=1 / 4, p_{1}=0.845, p_{2}=-p_{3}=0.362$, and $p_{4}=0.155$. The randomness is introduced in the respective order of the assigned weights $p_{i}$, at each step of the construction process [i.e., $\mu\left(A_{k_{1}, \ldots, k_{n}}\right)=p_{\sigma_{1}\left(k_{1}\right)} \cdots p_{\sigma_{n}\left(k_{n}\right)}$, where $\sigma_{1}, \ldots, \sigma_{n}$ are independent random permutations of the set $\{1, \ldots, s\}$ and correspond to the same law]. The values of the $\lambda_{i}$ have been chosen so that there is no gap in the support of $\mu$, i.e., Supp $\mu=A=[0,1]$. Furthermore, we have introduced a drift in our signal by superimposing the same $C^{\infty}$ perturbation $r(x)$ as the one used in Example 1: $f(x)=$ $s(x)+r(x)$ with $r(x)=R \sin (8 \pi x)$.

To estimate $\tau(q)$, we proceed as for Example 1, by computing the scaling behavior of the partition function $Z_{0, q}^{f}(a)$ over a significantly wide range of scales. Indeed, our measurement requires some averaging over several realizations of the random construction process described above. ${ }^{(32)}$ The results of this statistical analysis, performed with the analyzing wavelet $\psi^{(2)}$, are shown in Fig. 3b. The numerical data (circles) fall on a convex nonlinear curve which is particularly well fitted by the theoretical $\tau(q)$ spectrum (solid line). Its Legendre transform, $D(h)$, in Fig. 3c, is a singlehumped curve which is also in remarkable agreement, up to numerical uncertainties, with the theoretical singularity spectrum (solid line). The maximum of the $D(h)$ curve is found to be 1 , as expected for a function which is almost everywhere singular (cf. Section 4.4). Moreover, the range of Hölder exponents $\left[h_{\min }, h_{\max }\right]$ detected numerically matches the theoretical interval of singularities of $\mu,\left[\alpha_{\min }, \alpha_{\max }\right]$, where $\alpha_{\min }=\ln p_{1} / \ln \lambda_{1}=0.121 \ldots$ and $\alpha_{\max }=\ln p_{4} / \ln \lambda_{4}=1.345 \ldots$. Let us mention at this point that no "phase transition" phenomenon is observed for this class of signals when operating with different analyzing wavelets $\psi^{(N)}$.

Let us stress that for almost everywhere singular functions that are perturbed by an additional $C^{\infty}$ function, the local behavior of the wavelet coefficients is likely to be generally dominated by the power-law exponent governed by the singularities of $f$. Thus, provided the analyzing wavelets have a number of vanishing moments $N>h_{\max }$, the computation of the partition function $Z_{p, q}^{f}(a)$ will not be affected and consequently the singularity spectrum of the measures $D(h)$ will not be alterated. Signals having singularities distributed over the whole sampling interval are commonly encountered in various situations in applied sciences. ${ }^{(1,4-6,13,14)} \mathrm{We}$ refer the reader to our preliminary numerical study in refs. 31 and 32 , where our statistical approach based on wavelets has been successfully applied to fractional Brownian motions and to fully developed turbulent signals. 


\section{CONCLUSION}

To summarize, we have presented a first theoretical step toward a unified multifractal theory of singular distributions including singular measures and singular functions. The step performed here is a cautious one: we have only considered distribution functions of Bernoulli measures lying on disconnected Cantor sets invariant under some affine Markov maps, possibly perturbed by $C^{\infty}$ contributions. Our proofs have been established under specific hypotheses concerning the self-affine function under study as well as the shape of the analyzing wavelets. But our results are likely to remain valid under less stringent conditions. In particular, the hypothesis (H1) for the analyzing wavelet to have a compact support can be relaxed into an algebraic decay $\psi(x)=O\left(x^{-k}\right), k>0$, but at the expense of some (unnecessary) complications in the proofs. On the other hand, the results of some numerical applications strongly suggest that one should be able to extend our rigorous study to distribution functions of more general measures, e.g., the invariant measures of nonlinear expanding Markov maps including measures lying on a nonlacunar sets, real-valued measures that possesses a recursive structure, and the invariant measures of some well-known nonhyperbolic one-dimensional mappings (period-doubling Cantor set and the critical golden mean quasiperiodic trajectories). Moreover, we expect our theoretical results to apply to more general selfaffine functions, such as the realizations of some stochastic processes. Preliminary investigations in this context indicate that fractional Brownian motions ${ }^{(55)}$ are likely to be amenable to such a rigorous treatment relying on the wavelet decomposition.

This mathematical study provides algorithms for determining the $D(h)$ singularity directly from the considered self-affine function. Preliminary results reported in refs. 31 and 32 of an analysis of a fully developed turbulent velocity signal show that this method is readily applicable to experimental situations. Applications of this wavelet approach to turbulent dynamics in fractal growth phenomena, critical fluctuations in colloidal systems, and DNA "walk" nucleotide sequences are currently in progress. We believe that this method of determination of the singularity spectrum of fractal signals is likely to become as useful as the well-known phaseportrait reconstruction, Poincaré section, and first-return-map techniques for the analysis of chaotic time series.

\section{APPENDIX A}

We reproduce here the proof of the following lemma that one can find in ref. 40: 
Lemma 3. Let $f: \mathbb{R}^{+} \rightarrow \mathbb{R}^{+}$that satisfies $\exists s \in \mathbb{N}, \exists\left(c_{1}, \ldots, c_{s}\right) \in\left(\mathbb{R}^{+*}\right)^{s}$, $\left.\left.\exists\left(\lambda_{1}, \ldots, \lambda_{s}\right) \in\right] 0,1\right]^{s}$, and $\exists g>0$ so that

$$
0<a<g \Rightarrow f(a)=\sum_{k=1}^{s} c_{k} f\left(\frac{a}{\hat{\lambda}_{k}}\right)
$$

Then the two following relations hold:

(i) If $\forall \varepsilon, 0<\varepsilon<g, \sup _{[\varepsilon, g]} f(x)<+\infty$, then

$$
\sum_{k} c_{k}<1 \Leftrightarrow \lim _{a \rightarrow 0^{+}} f(a)=0
$$

(ii) If $\forall \varepsilon, 0<\varepsilon<g, \inf _{[\varepsilon, g]} f(x)>0$, then

$$
\sum_{k} c_{k}>1 \Leftrightarrow \lim _{a \rightarrow 0^{+}} f(a)=+\infty
$$

Proof. We will only prove the first part. The proof for the second part is along the same lines. Let $f^{+}: \mathbb{R}^{+} \rightarrow \mathbb{R}^{+}$be the function that satisfies the following conditions:

1. $0<a<g \Rightarrow f^{+}(a) \geqslant f(a)$.

2. $f^{+}$is monotonic.

3. If $v: \mathbb{R}^{+} \rightarrow \mathbb{R}^{+}$satisfies conditions 1 and 2 , then $v(a) \geqslant f^{+}(a)$ for $0<a<g$, i.e., $f^{+}$is the smallest monotonic function majorizing $f$. For $a \in] 0, g]$ it is explicitly given by one of the following expressions:

(a) If limsup $a \rightarrow 0^{+} f(a)<\infty$, then $f^{+}(a)=\sup _{y \in[0, a]} f(y)$.

(b) If $\limsup _{a \rightarrow 0^{+}} f(a)=\infty$, then $f^{+}(a)=\sup _{y \in[a, g]} f(y)$.

In the following $\Lambda_{\max }$ will denote $\sup _{i}\left\{\lambda_{i}\right\}$ and $\Lambda_{\min }$ will denote $\inf _{i}\left\{\lambda_{i}\right\}$. Let us suppose that $f^{\prime+}$ is another function satisfying the conditions $1-3$, but with a constant $g^{\prime}, 0<g^{\prime}<g$. Then let us prove that there exists $\eta>0$ such that

$$
f^{\prime+}(a)=f^{+}(a) \quad \text { for } \quad a<\eta
$$

In the case where $f^{+}$is given by (a), this is obvious. Suppose now that $f^{+}$ is given by the second expression (b). Then $f^{\prime+}$ is also obtained by a similar formula. For $0<a<g^{\prime}$ we have

$$
\begin{aligned}
f^{+}(a)= & \sup _{y \in[a, g]} f(y)=\max \left(\sup _{y \in\left[a, g^{\prime}\right]} f(y), \sup _{y \in\left[g^{\prime}, g\right]} f(y)\right) \\
& =\max \left(f^{\prime+}(a), \text { cst }\right)
\end{aligned}
$$

and therefore, since by hypothesis $f^{+}(a) \rightarrow \infty$ monotonically as $a$ goes to 0 , Eq. (A2) holds for $a$ small enough.

From the hypothesis that $f$ is bounded away from $\infty$ on any closed 
subset of $] 0, g]$, it follows that $f^{+}(a)<\infty$ for $\left.\left.a \in\right] 0, g\right]$. Using the relation (A1) and the fact that $f^{+}$majorizes $f$, we may write, for $0<a<g \Lambda_{\min }$,

$$
f(a) \leqslant \sum_{k=1}^{s} \mathfrak{c}_{k} f^{+}\left(\frac{a}{\lambda_{k}}\right)
$$

The right-hand side of this inequality is the sum of monotonic functions; it is thus a monotonic function. It majorizes $f$ on $\left.] 0, g A_{\min }\right]$. Therefore, since $f^{+}$is extremal [condition 3 and Eq. (A2)], it follows that for $a$ small enough, $f^{+}$satisfies the same type of equation:

$$
\begin{aligned}
f^{+}(a) & \leqslant \sum_{k=1}^{s} c_{k} f^{+}\left(\frac{a}{\lambda_{k}}\right) \\
& \leqslant\left(\sum_{k=1}^{s} c_{k}\right) \max \left(f^{+}\left(\frac{a}{\Lambda_{\min }}\right), f^{+}\left(\frac{a}{\Lambda_{\max }}\right)\right)
\end{aligned}
$$

where we have used the monotonicity of $f^{+}$( $a$ small enough). Since $\sum_{k=1}^{s} c_{k}<1$, it follows from $0<A_{\min } \leqslant \Lambda_{\max } \leqslant 1$ that

$$
\lim _{a \rightarrow 0^{+}} f^{+}(a)=\lim _{a \rightarrow 0^{+}} f(a)=0
$$

The proof for the case $\sum_{k=1}^{s} c_{k}>1$ is similar. We only have to replace $f^{+}$ by $f^{-}$, i.e., the maximal monotonic function that minorizes $f$, and to replace the majorizations by minorizations.

\section{APPENDIX B}

We want to prove the following lemma (cf. Section 4.3).

Lemma 8. Let $l \in \mathscr{L}_{f}$ and let $b(t)$ and $\left.\left.a(t)(t \in] 0,1\right]\right)$ be two continuous functions such that $\{(b(t), a(t))\}_{t \in j 0,1\}} \subset l$ and $\lim _{t \rightarrow 0} a(t)=0$. Then, $\exists b_{l} \in \mathbb{R}$ such that $\lim _{t \rightarrow 0} b(t)=b_{l}$.

Proof. Let us suppose that the function $b(t)$ does not converge when $t$ goes to 0 , i.e.,

$$
\left.\left.\left.\exists \varepsilon \in \mathbb{R}^{+*}, \quad \forall t_{0} \in\right] 0,1\right], \quad \exists\left(t_{1}, t_{2}\right) \in\right] 0, t_{0}\left[{ }^{2} \quad\left|b\left(t_{1}\right)-b\left(t_{2}\right)\right|>\varepsilon\right.
$$

Since $a(t)$ is $C^{0}$ and goes to 0 when $t \rightarrow 0$, then Eq. (B1) can be rewritten as follows:

$$
\begin{gathered}
\left.\left.\left.\exists \varepsilon \in \mathbb{R}^{+*}, \quad \forall \eta, \exists t_{0} \in\right] 0,1\right], \quad \exists\left(t_{1}, t_{2}\right) \in\right] 0, t_{0}\left[^{2}\right. \\
\text { such that }\left\{\begin{array}{l}
\left|b\left(t_{1}\right)-b\left(t_{2}\right)\right|>\varepsilon \\
\forall t \in] t_{1}, t_{2}[,|a(t)|<\eta
\end{array}\right.
\end{gathered}
$$

Then, it is clear that there exists an $n$ (large enough) so that the maxima 
line $l$ is going to cross an infinite number of times (and at smaller and smaller scales) at least one of the "holes" of the set

$$
\mathscr{A}^{(n)}=\bigcup_{\substack{k_{i}=1 \ldots s \\ i=1 \ldots n}} A_{k_{1}, \ldots, k_{n}}
$$

Moreover, if $b$ belongs to this hole and if $a$ is small enough, then $T_{\psi}[s](b, a)=0$ (because $\psi$ has a compact support) and therefore $T_{\psi}[f](b, a)=T_{\psi}[r](b, a)$. In other words, if $I=\left[b_{1}, b_{2}\right]$ is the interval representing this hole, then $\forall b \in I$, there exists a series $\left(a_{k}\right)_{k \in \mathbb{N}}$ (going to 0 ) so that $\forall k,\left(b, a_{k}\right) \in l$ and $T_{\psi}[f]\left(b, a_{k}\right)=T_{\psi}[r]\left(b, a_{k}\right)$. Since $\left(b, a_{k}\right)$ is a modulus maxima, then $\partial T_{\psi}[r] / \partial b\left(b, a_{k}\right)=0$. It follows that

$$
\int \psi^{\prime}\left(\frac{x-b}{a_{k}}\right) r(x) d x=0
$$

which is equivalent to

$$
\int \psi^{\prime}(x) r\left(b+a_{k} x\right) d x=0
$$

By replacing $r\left(b+a_{k} x\right)$ by its Taylor series at the order $N+1$, we get

$$
\sum_{p=0}^{N+1} \int \psi^{\prime}(x) \frac{\left(a_{k} x\right)^{p}}{p !} r^{(p)}(b) d x+o\left(a_{k}^{N+1}\right)=0
$$

Moreover, by using the hypothesis (H5), it follows that $\int x^{k} \psi^{\prime}(x) d x=0$ for $0 \leqslant k<N+1$ and $\int \psi^{\prime}(x) x^{N+1} \neq 0$; therefore Eq. (B3) becomes

$$
a_{k}^{N+1}\left(r^{(N+1)}(b) \int \psi^{\prime}(x) x^{N+1} d x+o(1)\right)=0
$$

which implies that $r^{(N+1)}(b)=0$. It follows that $\left.r^{(N+1)}\right|_{I}=0$; therefore $\left.r\right|_{I}$ is a polynomial of order $N$ and then, for small enough $a$, $\left.T_{\psi}[f](\cdot, a)\right|_{l}=$ Cte. This last result contradicts the fact that the $\left\{\left(b, a_{k}\right)\right\}_{k}$ are modulus maxima of $T_{\psi}$.

\section{APPENDIX C}

We want to prove the following lemma (cf. Section 4.3).

Lemma 9. Let us suppose that:

(H6) $N>\max \left(\alpha_{\max }, 3 \alpha_{\max } / 2-1\right)$ [cf. (H5) for the definition of $N$ and Lemma 6 for the definition of $\left.\alpha_{\max }\right]$.

(H7) $d\left(\partial T_{\psi}[s] / \partial b\right)=\left(\partial^{2} T_{\psi}[s] / \partial b^{2}\right) d b+\left(\hat{\partial}^{2} T_{\psi}[s] / \partial a \partial b\right) d a \neq 0$ on any maxima line in $\mathscr{L}_{s}$.

(H8) $\partial^{2} T_{\psi}[s] / \partial b^{2} \neq 0$ on any maxima line in $\mathscr{L}_{s}$ except at its boundary points. 
Then for all $\varepsilon>0$, there exists $a_{0}>0, k>1$, and $C>0$ such that

$\forall a<a_{0}, \quad \forall l \in \mathscr{L}_{s}(a), \quad a_{l}>a /(1-\varepsilon) \quad$ then $\quad \exists ! l_{1} \in \mathscr{L}_{f}(a)$ such that

$$
\forall\left(x, a^{\prime}\right) \in l, \quad \exists !\left(x_{1}, a^{\prime}\right) \in l_{1}, \quad\left|x_{1}-x\right|<C a^{\prime k}
$$

Moreover,

$$
T_{\psi}[f]\left(x_{1}, a^{\prime}\right)=T_{\psi}[s]\left(x, a^{\prime}\right)\left[1+\eta\left(x, a^{\prime}\right)\right]
$$

where $\eta\left(x, a^{\prime}\right)$ is a function which is uniformly bounded by a decreasing function $F\left(a^{\prime}\right)$ (independent of $l$ ) which goes to 0 when $a^{\prime}$ goes to 0 .

Proof. (H7) mainly means that the maxima lines of $T_{\psi}[s]$ do not "bifurcate"; it implies that the distance between two modulus maxima (belonging to two different maxima lines) at the same scale $a$ (with $a$ varying from $\Lambda g$ to $g$ ) is bounded away from 0 . Then, by using the selfsimilarity property [Lemma 1(ii)], it follows that there exists a constant $C>0$ so that the minimum distance between two maxima lines at scale $a>0$ is greater than $C a$ (for the sake of simplicity we will suppose that $C=1$ ). Then, if $l \in \mathscr{L}_{s}$ and $k>1$, we define the following neighborhood of $l$ :

$$
\mathscr{V}_{k}(l)=\left\{\left(x^{\prime}, a\right), \exists(x, a) \in l /\left|x-x^{\prime}\right|<a^{k}\right\}
$$

By definition, $\mathscr{V}_{k}(l)$ does not contain any modulus maximum belonging to another maxima line.

We fix $\varepsilon>0$ ( $\varepsilon$ small). By the definition of a modulus maximum (see Definition 1), $T_{\psi}[s]$ does not vanish on any maxima line in $\mathscr{L}_{s}$. Then there exists $C_{1}>0$ such that for all $l \in \mathscr{L}_{s}(a)$ (with $A g \leqslant a \leqslant g$ ) with $a_{l}>a /(1-\varepsilon)$, then

$$
\left|T_{\psi}[s]\right|>C_{1} \quad \text { in a neighborhood of } l
$$

Furthermore, by using the self-similarity property [Lemma 1(ii)], one can prove (in the same way in which we proved Lemmas 5 and 6 ) that there exists $a_{1}>0$ such that

$$
\begin{gathered}
\forall a<a_{1}, \quad \forall l \in \mathscr{L}_{s}(a) \text { such that } a_{l}>a /(1-\varepsilon) \text { then } \\
\left(x, a^{\prime}\right) \in \mathscr{V}_{k}(l) \Rightarrow\left|T_{\psi}[s]\left(x, a^{\prime}\right)\right|>C_{1} a^{\prime \alpha_{\max }}
\end{gathered}
$$

Similarly, one can prove [from (H8)] that there exists $C_{2}>0$ and $a_{2}>0$ such that

$$
\begin{gathered}
\forall a<a_{2}, \quad \forall l \in \mathscr{L}_{s}(a) \quad \text { such that } \quad a_{l}>a /(1-\varepsilon) \quad \text { then } \\
\left(x, a^{\prime}\right) \in \mathscr{V}_{k}(l) \Rightarrow\left|\frac{\partial^{2} T_{\psi}[s]}{\partial b^{2}}\left(x, a^{\prime}\right)\right|>C_{2} a^{\prime \alpha_{\max }-2}
\end{gathered}
$$


Let $a_{0}=\min \left(a_{1}, a_{2}\right)$. From now we will suppose that $a<a_{0}, l \in \mathscr{L}_{s}(a)$, $a_{l}>a /(1-\varepsilon)$. Moreover, we choose $k=1+k_{1}$ with $\alpha_{\max } / 2<k_{1}<$ $N-\alpha_{\max }+1$ (such a $k_{1}$ exists because $N>3 \alpha_{\max } / 2-1$ ). We then consider $\left(x, a^{\prime}\right) \in l$ and we want to prove that there exists a unique modulus maximum of $T_{\psi}[f]$ at a point $\left(x_{1}, a^{\prime}\right) \in \mathscr{V}_{k}(l)$. Let us suppose that $T_{\psi}[s]$ is negative and $\partial^{2} T_{\psi}[s] / \partial b^{2}$ positive in $\mathscr{V}_{k}(l)$ (the other case, corresponding to $T_{\psi}[s]$ positive and $\partial^{2} T_{\psi}[s] / \partial b^{2}$ negative, can be solved in the same way).

Then, we are looking for a point $x(\lambda)=\left(x+\lambda, a^{\prime}\right)$ (with $\left.|\hat{\lambda}|<a^{k}\right)$ which would be a maximum of $T_{\psi}[f]$. From Lemma 7 , one can easily prove that there exist two functions $R_{1}$ and $R_{2}$ (uniformly bounded by a constant $C$ ) so that

$$
\frac{\partial T_{\psi}[f]}{\partial b}(x(\lambda))=\frac{\partial T_{\psi}[s]}{\partial b}(x(\lambda))+a^{\prime N} R_{1}(x(\lambda))
$$

and

$$
\frac{\partial^{2} T_{\psi}[f]}{\partial b^{2}}(x(\lambda))=\frac{\partial^{2} T_{\psi}[s]}{\partial b^{2}}(x(\lambda))+a^{\prime N} R_{2}(x(\lambda))
$$

We are looking for $\lambda$ such that $d(\lambda)=\partial T_{\psi}[f] / \partial b(x(\lambda))=0$. From Eqs. (C2) and (C4), we get that

$$
d^{\prime}(\lambda)>C_{2} a^{\prime \alpha_{\max }-2}-C a^{\prime N}>0
$$

$\left(R_{1}\right.$ and $R_{2}$ are defined independently of $l$. So we can assume that we have chosen $a_{0}$ small enough so that $C_{2} a^{\alpha_{\max }-2}-C a^{N}>0$.) Then $d$ is a strictly increasing function. On the other hand, if $\lambda>0$, it follows from Eq. (C2) that

$$
\frac{\partial T_{\psi}[s]}{\partial b}(x(\lambda))>C_{2} \lambda a^{\prime \alpha_{\max }-2}
$$

and therefore, using Eq. (C3),

$$
d(\lambda)>C_{2} \lambda a^{\prime \alpha_{\max }-2}-C a^{\prime N}
$$

One thus gets

$$
d\left(a^{\prime 1+k_{1}}\right)>C_{2} a^{\prime k_{1}+\alpha_{\max }-1}-C a^{\prime N}>0
$$

(since $k_{1}<N-\alpha_{\max }+1$ we can suppose that we have chosen $a_{0}$ small enough so that the right-hand side is strictly positive). In the same way we get

$$
d\left(-a^{\prime+k_{1}}\right)<0
$$


Therefore, $d$ is an increasing function which goes [when $x(\lambda)$ varies in $\left.\mathscr{V}_{k}(l)\right]$ from negative values to positive values; it has one single zero: $\lambda=\lambda\left(x, a^{\prime}\right)$. As $d$ is strictly increasing, this zero corresponds to a point where $T_{\psi}[f]$ is an extremum (i.e., the second derivative of $T_{\psi}$ with respect to $b$ is not 0$)$. Let us determine the sign of $T_{\psi}[f]$ at the point $\left(x+\lambda\left(x, a^{\prime}\right), a^{\prime}\right)$. The maximum absolute value of the second derivative is uniformly smaller than $C^{\prime} a^{\prime-2}$ (where $C^{\prime}$ is a uniform upper bound of $\left.T_{\psi}[s]\right)$; then as $\left(x+\lambda, a^{\prime}\right) \in \mathscr{V}_{k}(l)$ we get

$$
\begin{aligned}
T_{\psi}[f]\left(x+\lambda, a^{\prime}\right) & =T_{\psi}[s]\left(x, a^{\prime}\right)+\lambda^{2} \frac{\partial^{2} T_{\psi}[s]}{\partial b^{2}}\left(x+\lambda, a^{\prime}\right)+a^{\prime N} R\left(x+\lambda, a^{\prime}\right) \\
& <-C_{1} a^{\prime \alpha_{\max }}+C^{\prime} a^{\prime 2\left(1+k_{1}\right)} a^{\prime-2}+C^{\prime \prime} a^{\prime N} \\
& <-C_{1} a^{\prime \alpha_{\max }}+C^{\prime} a^{\prime 2 k_{1}}+C^{\prime \prime} a^{\prime N}
\end{aligned}
$$

where $C_{1}, C^{\prime}, C^{\prime \prime}$ are "global" constants. Moreover, as $k_{1}>\alpha_{\max } / 2$ and $N>\alpha_{\max }$ [see (H6)], we can choose $a_{0}$ small enough so that

$$
T_{\psi}[f]\left(x+\lambda, a^{\prime}\right)=T_{\psi}[s]\left(x, a^{\prime}\right)\left[1+\eta\left(x, a^{\prime}\right)\right]<0
$$

with

$$
\left|\eta\left(x, a^{\prime}\right)\right|<a^{\prime-\alpha_{\max }} O\left(a^{\prime 2 k_{1}}+a^{\prime N}\right)
$$

It follows that the modulus maximum $\left(x, a^{\prime}\right)$ of $T_{\psi}[s]$ has been "transformed" into the modulus maximum $\left(x+\lambda\left(x, a^{\prime}\right), a^{\prime}\right)$ of $T_{\psi}[f]$.

We still need to prove that the line $l$ is "transformed" to a set $l_{1}$ which is a continuous line. From (C5) we get

$$
\frac{\partial^{2} T_{\psi}[f]}{\partial b^{2}}\left(x+\lambda\left(x, a^{\prime}\right), a^{\prime}\right) \neq 0
$$

Then, by using the implicit function theorem, we know that around each modulus maximum $\left(x+\lambda\left(x, a^{\prime}\right), a^{\prime}\right)$ there exists a small line $\left[\in \mathscr{V}_{k}(l)\right]$ along which $\partial T_{\psi}[f] / \partial b$ is zero. Let us call $l_{1}$ the union of all these lines. Since we have the same control on $T_{\psi}[f]$ as we had on $T_{\psi}[s]$ [in terms of lower bounds of the consecutive derivatives in $\left.\mathscr{V}_{k}(l)\right], l_{1}$ is made of modulus maxima and is a close set relative to the set $\mathscr{V}_{k}(l)$. Moreover, if $k=1+k_{1}<k^{\prime}<1+N-\alpha_{\max }+1$, then at any scale $a^{\prime}, l_{1}$ is always at a distance greater than $a^{\prime k}-a^{\prime k^{\prime}}$ from the right and left borders of $\mathscr{V}_{k}(l)$. On the other hand, from Eq. (C8), it follows that a connected line of maxima cannot go down (to the small scales) and then up (to the large scales); this 
means that on such a line there is a unique modulus maximum at each scale. Thus, if we consider the longest connected line $l_{2} \subset l_{1}$, it is easy to prove that $l_{2}$ contains modulus maxima as close as we want to the scale 0 and that $l_{2}=l_{1}$.

\section{APPENDIX D}

We want to prove the following lemma (see Section 4.3).

Lemma 10. Let us suppose that:

(H9) If both $T_{\psi}[s]$ and $\partial T_{\psi}[s] / \partial b$ are vanishing at a certain point, then both $\partial T_{\psi}[s] / \partial a$ and $\partial^{2} T_{\psi}[s] / \partial b^{2}$ are nonzero.

Then $\exists \varepsilon, \exists a_{0}, \forall l \in \mathscr{L}_{s}(a), a_{l}>a /(1-\varepsilon)$,

$$
\text { if } a_{0}>a_{l}, \quad \text { then } a_{P(l)}<a_{l} /(1-\varepsilon)
$$

Proof. The proof uses very similar techniques to those used in Appendix $\mathrm{C}$, but it is much longer and not very interesting. Thus, we do not give the full proof, but just describe the main trends.

Let us first give two definitions:

(a) In the following, we will say that $(b, a)$ is an extremum of $T_{\psi}$ iff $\partial T_{\psi} / \partial b(b, a)=0$.

(b) Moreover, in the same way as in Definition 2, we will call an extremum line of $T_{\psi}$ any connected line made of extrema. [We define also $\mathscr{L}^{\text {ext }}(a)$ and $\mathscr{L}^{\text {ext }}$ as in Notation 1.]

As seen in Section 4.3 , a point $\left(x_{l}, a_{l}\right)$ where a maxima line first "appears" can be of two types (see 1 and 2 in Section 4.3). From (H9), it follows that these two types are exclusive:

(1) $T_{\psi}[s]\left(x_{l}, a_{l}\right)=0$. By the continuity of $\partial T_{\psi}[s] / \partial b$ and by the definition of $a_{l}$, it is clear that $\partial T_{\psi}[s] / \partial b\left(x_{l}, a_{l}\right)=0$ and that there exists an extrema line in $\mathscr{L}_{s}^{\text {ext }}\left(a_{l}\right)$ which contains $l$. Then, from $(\mathrm{H} 9)$, we get $\partial^{2} T_{\psi}[s] / \partial b^{2}\left(x_{l}, a_{l}\right) \neq 0$ and by using the implicit function theorem, it follows that there exists $\varepsilon$ small and $l^{\text {ext }} \in \mathscr{L}_{s}^{\text {ext }}\left(a_{l} /(1-\varepsilon)\right)$ such that $l \subset l^{\text {ext }}$ [let us note that the self-similarity property, Lemma 1(ii), allows us to choose $\varepsilon$ independently of $l]$. Indeed, the beginnings of the proofs in Appendix $\mathrm{C}$ [until Eq. (C6)] hold when applied to $l^{\text {ext }}$, and we thus get a perturbed extrema line $P^{\text {ext }}\left(l^{\text {ext }}\right) \in \mathscr{L}_{f}^{\text {ext }}\left(a_{l} /(1-\varepsilon)\right)\left[\right.$ with $\left.P(l) \subset P^{\text {ext }}\left(l^{\text {ext }}\right)\right]$. Moreover, we know [from (H9)] that $\partial T_{\psi}[s] / \partial a\left(x_{l}, a_{l}\right) \neq 0$ and therefore (for $\varepsilon$ small enough) $\left(x, a_{l} /(1-\varepsilon)\right) \in l^{\text {ext }}$ is not a modulus maximum of $T_{\psi}[s]$ (i.e., it is either a positive minimum or a negative maximum). For 
$a_{l}$ small enough, it is clear that this will still be true for the corresponding perturbed extremum $\left(x+\lambda\left(x, a_{l} /(1-\varepsilon)\right), a_{l} /(1-\varepsilon)\right) \in P^{\text {ext }}\left(l^{\text {ext }}\right)$. Thus, if $l_{1} \in \mathscr{L}_{f}, P(l) \subset l_{1}$, then $l_{1} \subset P^{\text {ext }}\left(l^{\text {ext }}\right)$ and $a_{P(l)} \leqslant a_{i} /(1-\varepsilon)$.

(2) $\partial^{2} T_{\psi}[s] / \partial b^{2}\left(x_{l}, a_{l}\right)=0$. As $\partial^{2} T_{\psi}[s] / \partial b \partial a\left(x_{l}, a_{l}\right) \neq 0[$ see (H7)], by using the implicit function theorem, one can show that there exists $\varepsilon$ (small and independent of $l$ ) and an extrema line $l^{\text {ext }}$ which contains the point $\left(x_{l}, a_{l}\right)$. Let us just consider the portion $l_{P}^{\text {ext }}$ of $l^{\text {ext }}$ which is around $\left(x_{l}, a_{l}\right)$ [i.e., $\left.\left\{(x, a) \in l^{\text {ext }}, a_{l}(1-\eta)<a<a_{l} /(1-\eta)\right\}\right]$. Then, in the same way as in the proof in Appendix $C$, but by exchanging the roles played by $a$ and $x$, we can associate to any extremum $(x, a) \in l_{P}^{\text {ext }}$ a unique perturbed extremum $(x, a+\lambda(a, x))$ with $|\lambda|<a^{k}, k>1$. Moreover, if $(x, a)$ is not a modulus maximum (but just an extremum), then, for $a_{l}$ small enough, the corresponding perturbed extremum is also not a modulus maximum. One can then prove that the perturbed portion remains "around" the scale $a_{l}$ and that it contains the end of $P(l)$ [i.e., $\left.\left(x_{P(l)}, a_{P(l)}\right)\right]$; thus, $a_{P(l)} \leqslant a_{l} /(1-\varepsilon)$.

\section{ACKNOWLEDGMENTS}

We thank F. Argoul, S. Jaffard, S. Mallat, and Y. Meyer for interesting and useful discussions. This work was supported by the Direction des Recherches Etudes et Techniques under contract DRET No. 89/196.

\section{REFERENCES}

1. B. B. Mandelbrot, The Fractal Geometry of Nature (Freeman, San Francisco, 1982).

2. T. C. Halsey, M. H. Jensen, L. P. Kadanoff, I. Procaccia, and B. I. Shraiman, Phys. Rev. A 33:1141 (1986).

3. G. Paladin and A. Vulpiani, Phys. Rep. 156:148 (1987).

4. H. E. Stanley and N. Ostrowski, eds., On Growth and Form: Fractal and Nonfractal Patterns in Physics (Martinus Nijhof, Dordrecht, 1986), and references therein; H. E. Stanley and N. Ostrowski, eds., Random Fluctuations and Pattern Growth (Kluwer Academic, Dordrecht, 1988), and references therein.

5. L. Pietronero and E. Tosatti, eds., Fractals in Physics (North-Holland, Amsterdam, 1986), and references therein.

6. A. Aharony and J. Feder, eds., Essays in honour of B. B. Mandelbrot, Fractals in Physics, Physica D 38 (1989), and references therein.

7. E. B. Vul, Ya. G. Sinai, and K. M. Khanin, Usp. Mat. Nauk 39:3 (1984) [J. Russ. Math. Surv. 39:1 (1984)].

8. R. Benzi, G. Paladin, G. Parisi, and A. Vulpiani, J. Phys. A 17:3521 (1984).

9. D. Rand, Ergodic Theory Dynamic Syst. 9:527 (1989).

10. P. Collet, J. Lebowitz, and A. Porzio, J. Stat. Phys. 47:609 (1987).

11. M. J. Feigenbaum, J. Stat. Phys. 46:919, 925 (1987).

12. R. Badii, Thesis, University of Zurich (1987). 
13. J. Feder, Fractals (Pergamon, New York, 1988), and references therein.

14. T. Vicsek, Fractal Growth Phenomena (World Scientific, Singapore, 1989), and references therein.

15. P. Meakin, in Phase Transition and Critical Phenomena, Vol. 12, C. Domb and J. L. Lebowitz, eds. (Academic Press, Orlando, Florida, 1988).

16. B. Mandelbrot, J. Fluid Mech. 62:331 (1974).

17. U. Frisch, P. L. Sulem, and M. Nelkin, J. Fluid Mech. 87:719 (1978).

18. C. Meneveau and K. R. Sreenivasan, J. Fluid Mech. 224:429 (1991).

19. P. Grassberger, Phys. Lett. A 97:227 (1983).

20. H. G. E. Hentschel and I. Procaccia, Physica D 8:435 (1983).

21. P. Grassberger and I. Procaccia, Physica D 13:34 (1984).

22. Ya. G. Sinai, Usp. Mat. Nauk 27:21 (1972) [J. Russ. Math. Surv. 166:21 (1972)].

23. R. Bowen, Lecture Notes in Mathematics, Vol. 470 (Springer, New York, 1975).

24. D. Ruelle, Statistical Mechanics (Addison-Wesley, Reading, Massachusetts, 1969); Thermodynamics Formalism (Addison-Wesley, Reading, Massachusetts, 1978).

25. T. Bohr and T. Tèl, in Direction in Chaos, Vol. 2, Hao Bai-Lin, ed. (World Scientific, Singapore, 1988).

26. U. Frisch and G. Parisi, Fully developed turbulence and intermittency, in Proceedings of International School on Turbulence and Predictability in Geophysical Fluid Dynamics and Climate Dynamics, M. Ghil, R. Benzi, and G. Parisi, eds. (North-Holland, Amsterdam, 1985), p. 84.

27. A. L. Barabási and T. Vicsek, Phys. Rev. A 44:2730 (1991).

28. F. Anselmet, Y. Gagne, E. J. Hopfinger, and R. A. Antonia, J. Fluid Mech. 140:63 (1984).

29. Y. Gagne, E. J. Hopfinger, and U. Frisch, in New Trends in Nonlinear Dynamics and Pattern Forming Phenomena: The Geometry of Nonequilibrium, P. Huerre and P. Coullet, eds. (Plenum Press, New York, 1988).

30. B. Castaing, Y. Gagne, and E. J. Hopfinger, Physica D 46:177 (1990).

31. J. F. Muzy, E. Bacry, and A. Arnéodo, Phys. Rev. Lett. 67:3515 (1991).

32. A. Arnéodo, E. Bacry, and J. F. Muzy, Wavelet analysis of fractal signals: Direct determination of the singularity spectrum of fully developed turbulence data (Springer-Verlag, Berlin, 1991), to appear.

33. J. F. Muzy, E. Bacry, and A. Arnéodo, Multifractal formalism for self-affine signals: The structure function approach versus the wavelet transform modulus maxima method, Phys. Rev. E, to appear.

34. A. Grossmann and J. Morlet, SIAM J. Math. Anal. 15:723 (1984).

35. I. Daubechies, A. Grossmann, and Y. Meyer, J. Math. Phys. 127:1271 (1986).

36. J. M. Combes, A. Grossmann, and P. Tchamitchian, eds., Wavelets (Springer-Verlag, Berlin, 1988), and references therein.

37. Y. Meyer, Ondelettes (Hermann, Paris, 1990).

38. P. G. Lemarié, ed., Les Ondelettes en 1989 (Springer-Verlag, Berlin, 1990).

39. A. Arnéodo, G. Grasseau, and M. Holschneider, Phys. Rev. Lett. 61:2281 (1988); and in Wavelets, J. M. Combes, A. Grossmann, and P. Tchamitchian, eds. (Springer-Verlag, Berlin, 1988), p. 182.

40. M. Holschneider, J. Stat. Phys. 50:963 (1988); Thesis, University of Aix-Marseille II (1988).

41. A. Arnéodo, F. Argoul, J. Elezgaray, and G. Grasseau, in Nonlinear Dynamics, G. Turchetti, ed. (World Scientific, Singapore, 1988), p. 130.

42. A. Arnéodo, F. Argoul, and G. Grasseau, in Les Ondelettes en 1989, P. G. Lemarié, ed. (Springer-Verlag, Berlin, 1990), p. 125.

43. G. Grasseau, Thesis, University of Bordeaux (1989). 
44. A. Arnéodo, F. Argoul, E. Bacry, J. Elezgaray, E. Freysz, G. Grasseau, J. F. Muzy, and B. Pouligny, in Wavelets and Their Applications, Y. Meyer, ed. (Springer, Berlin, 1992), p. 286.

45. M. Holschneider and P. Tchamitchian, in Les Ondelettes en 1989, P. G. Lemarié, ed. (Springer-Verlag, Berlin, 1990), p. 102.

46. S. Jaffard, C. R. Acad. Sci. Paris 308:79 (1989); Sur la dimension de Hausdorff de points singuliers d'une fonction, preprint (1991).

47. S. Mallat and W. L. Hwang, IEEE Trans. on Information Theory 38:617 (1992).

48. P. Cvitanovic, in XV International Colloquium on Group Theoretical Methods in Physics, R. Gilmore, ed. (World Scientific, Singapore, 1987).

49. P. Grassberger, R. Badii, and A. Politi, J. Stat. Phys. 51:135 (1988).

50. D. Katzen and I. Procaccia, Phys. Rev. Lett. 58:1169 (1987).

51. T. Bohr and M. H. Jensen, Phys. Rev. A 36:4904 (1987).

52. A. Arnéodo, F. Argoul, E. Freysz, J. F. Muzy, and B. Pouligny, in Wavelet and Their Applications, M. B. Ruskai, G. Beylkin, R. Coifman, I. Daubechies, S. Mallat, Y. Meyer, and L. Raphael, eds. (Jones and Bartlett, Boston, 1991), p. 241.

53. J. M. Ghez and S. Vaienti, Nonlinearity 5:772; 791 (1992).

54. K. Falconer, The Geometry of Fractal Sets (Cambridge University Press, Cambridge, 1985).

55. P. Flandrin, Wavelet analysis and synthesis of fractional Brownian motion, preprint (1991). 